\title{
Adjustable vertical vibration isolator with a variable ellipse curve mechanism
}

\author{
Takehiko Asai $^{1 *}$, Yoshikazu Araki ${ }^{2}$, Kosuke Kimura $^{3}$ and Takeshi Masui ${ }^{4}$ \\ ${ }^{1}$ Faculty of Engineering, Information and Systems, University of Tsukuba, Tsukuba, Ibaraki 305-8573, Japan \\ ${ }^{2}$ Department of Architecture and Architectural Engineering, Kyoto University, Nishikyo, Kyoto 615-8540, Japan \\ ${ }^{3}$ Geo-Graphic Design Lab., Nakamiya, Osaka 535-0003, Japan \\ ${ }^{4}$ Department of Architecture, Kansai University, Yamatecho, Suita, Osaka 564-8680, Japan
}

\section{SUMMARY}

This paper presents a passive vertical quasi-zero-stiffness (QZS) vibration isolator intended for relatively small objects. The present isolator have features of compactness, long stroke, and adjustability to various load capabilities. To realize these features, we use constant-force springs, which sustain constant load regardless of their elongation and propose a variable ellipse curve mechanism which is inspired by the principle of ellipsographs. The variable ellipse curve mechanism can convert the restoring force of the horizontally placed constant-force springs to the vertical restoring force of the vibration isolator. At the same time as converting the direction, the vertical restoring force can be adjusted by changing the ratio of the semi-minor axis to the semi-major one of the ellipse. In this study, a prototype of a class of QZS vibration isolator with the proposed variable ellipse curve mechanism is created. Shaking table tests are performed to demonstrate the efficacy of the present mechanism, where the prototype is subjected to various sinusoidal and earthquake ground motions. It is demonstrated through the shaking table tests that the prototype can reduce the response acceleration within the same specified tolerance even when the mass of the vibration isolated object is changed. Copyright (C) 2017 John Wiley \& Sons, Ltd.

Received ...

KEY WORDS: vertical vibration isolator, quasi-zero-stiffness, shaking table test, ellipsograph

\section{INTRODUCTION}

Recently, many earthquakes whose up-down (UD) component of acceleration is almost to $1 \mathrm{~g}$ or more have been recorded. To protect fragile objects such as precision instruments and fine arts from these intense ground motions, needs for not only horizontal vibration isolators but also vertical ones have been growing $[1,2]$. Therefore this paper aims to propose a passive vertical vibration isolator which can be combined with commercially available horizontal isolators or which can be used in horizontally isolated buildings.

The practical use of horizontal isolators has been already achieved, while vertical ones have not yet reached as a maturity level as horizontal ones have. This is because, in the design of passive vertical vibration isolators, excessive deformation due to gravity force is one of the main obstacles. To overcome the difficulty, considerable effort has been devoted to research on quasi-zero-stiffness (QZS) vibration isolators $[3,4,5,6,7,8,9,10]$. However, most vertical isolators in the past do not realize long stroke, large loading capacity, and adjustability all at once with simple mechanism.

QZS vibration isolators are designed so that their restoring forces satisfy the following two conditions: (1) initial stiffness, which resists self-weight, is large, and (2) the tangent stiffness,

\footnotetext{
${ }^{*}$ Correspondence to: Faculty of Engineering, Information and Systems, University of Tsukuba, Tsukuba, Ibaraki 3058573, Japan. E-mail: asai@kz.tsukuba.ac.jp 
(a)

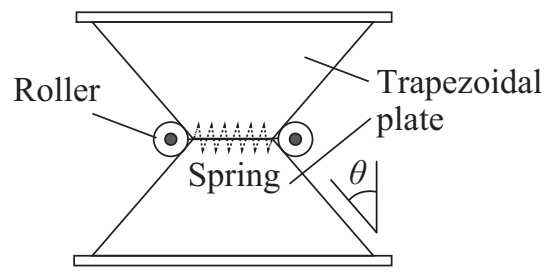

(b)

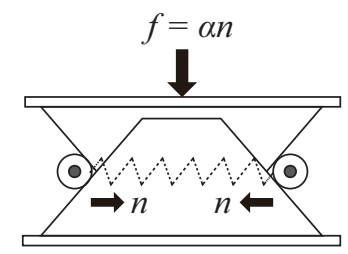

Figure 1. Vertical vibration isolator with the vertical amplification mechanism

which resists dynamic load, is close to zero around the static equilibrium position. So far, the authors proposed simple QZS mechanisms satisfying these two conditions by using constant-force springs, which sustain constant load regardless of their elongation [11]. Also the authors developed a horizontal isolator with constant-force springs and by combining the vertical and horizontal isolators, a 2-D (vertical and horizontal) isolator was proposed. Then through shaking table tests, how horizontal vibration affects to a tall object was investigated [12]. The loading capacity of a single constant-force spring is, however, limited to the order of tens to hundreds newton while the size of a constant-force spring is relatively large. To achieve large loading capacity in QZS mechanisms, e.g., several to tens kilonewton, a number of constant-force springs are necessary, which makes the size of QZS mechanisms unnecessarily large. This limits the applicability of QZS vibration isolators with constant-force springs to light weight applications.

To increase the limit of the load capability of the device, the authors proposed a device with an amplification mechanism [13]. The basic idea of the device lies in converting the horizontal restoring force of the constant-force springs to the vertical direction. A simple example of this mechanism can be realized by vertically placed trapezoidal plates shown in Fig. 1. This device converts the restoring force of the constant-force springs $n$ to the restoring force of the isolator $f$. Suppose the angle of the plate is $\theta$ as shown in the figure, then the ratio of $f$ to $n$ can be expressed as

$$
\frac{f}{n}=\tan \theta=\alpha(\theta)
$$

This equation indicates that the restoring force of the isolator is obtained by the restoring force of the springs $n$ times $\tan \theta$. Then the restoring force can be adjusted by changing the angle $\theta$. Thus $\alpha$ can be called amplification factor.

In this mechanism, the amplification factor $\alpha$ is a function of the angle of trapezoidal plate $\theta$. Hence, the restoring force of the isolator can be adjustable by changing $\theta$. The authors realized this variable mechanism by using cranks and a screw jack for the vertical vibration isolator and verified its effectiveness through shaking table tests and numerical simulations [13]. Also, the similar mechanism was realized by using superelastic Cu-Al-Mn shape memory alloy bars instead of constant-force springs [14].

However, in these studies, the force directions are converted by the vertically placed trapezoidal plates. Thus, physically, the device cannot be lower than the height of the trapezoidal plate. Hence the height and stroke of the isolator are restricted. Especially, in isolators, the lack of stroke is a serious problem because it induces a risk of collisions which cause excessive response accelerations. To address this issue, this paper proposes a horizontal amplification mechanism which makes the device more compact and ensures enough stroke. In addition, the mechanism proposed in this paper enables the isolator to adjust to various weight without complicated procedures.

In this paper, firstly, an amplification mechanism using a horizontally placed ellipse curve plate is introduced. Then the relationship between the shape of the ellipse curve and the produced restoring force is investigated. Secondly, to adapt to various weight of the target objects, a simple mechanism based on the principle of ellipsographs which can change the trajectory of the ellipse curve is proposed. And the adjustable vertical vibration isolator with this mechanism is presented. Then a prototype of the proposed adjustable vertical vibration isolator is made, which realizes almost twice as long stroke as the isolator presented by the authors in the literature [13] under the condition 


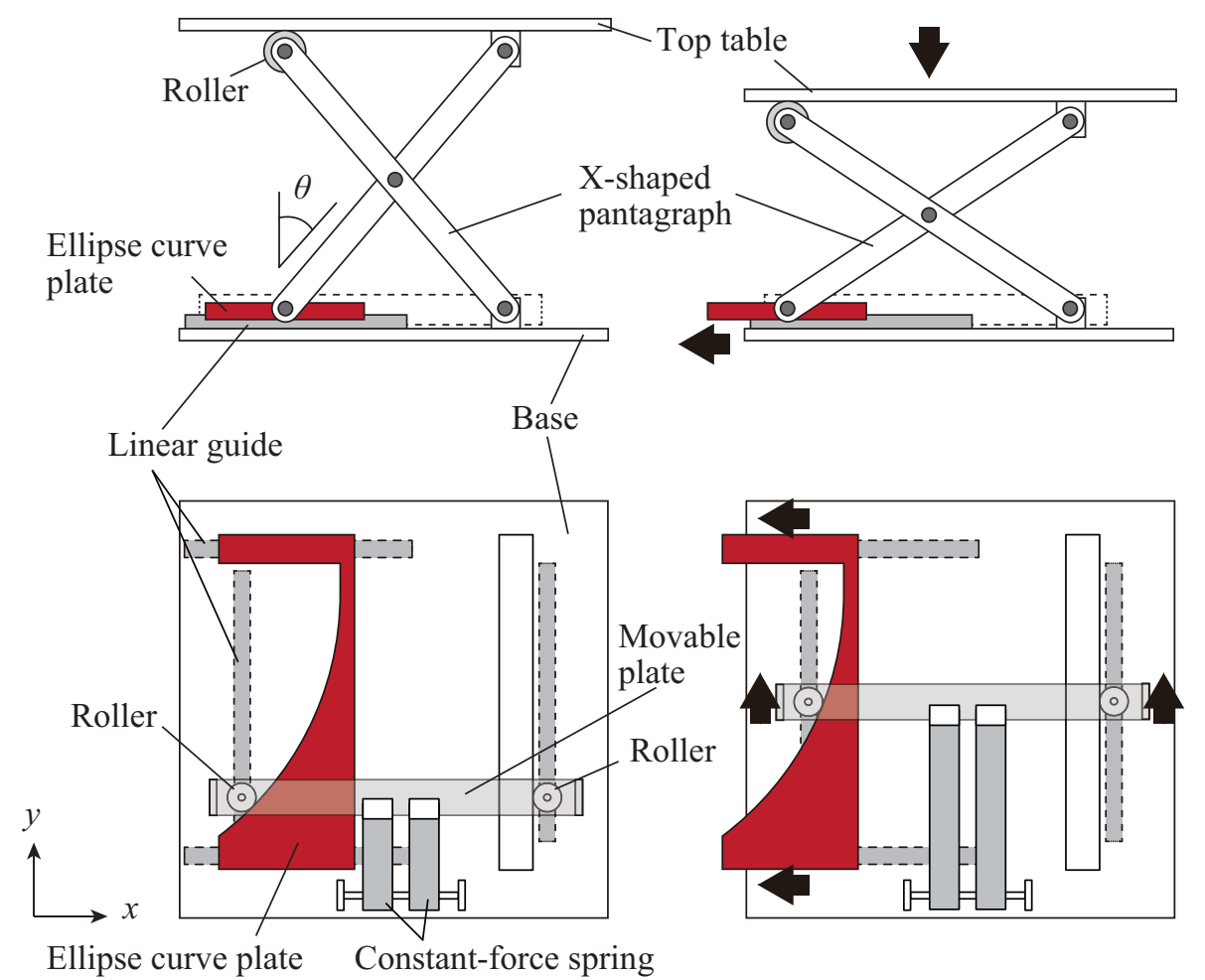

Figure 2. Horizontal amplification mechanism

of the same height. Subsequently, shaking table tests and numerical simulations are conducted. Conclusions obtained from this study then follow.

\section{HORIZONTAL AMPLIFICATION MECHANISM USING AN ELLIPSE CURVE PLATE}

In this section, we introduce a horizontal amplification mechanism using an ellipse curve and its advantages over the vertical amplification mechanism proposed in the literature [13, 14]. Then the restoring force produced by this mechanism is examined.

\subsection{Horizontal amplification mechanism}

The horizontal amplification mechanism using an ellipse curve is illustrated in Fig. 2 in two heights. As shown, this mechanism is designed to interlock three parts, i.e., a movable plate, an ellipse curve plate, and two pairs of X-shaped pantagraphs. The movable plate is connected to the constant-force springs which are fixed to the base. The movable plate is constrained to slide only in the $y$-direction by linear guides. The ellipse curve plate is allowed to move in the $x$-direction by linear guides as well. One of the rollers attached to the movable plate contacts on the side surface of the ellipse curve plate to transmit the restoring force from the springs to the plate. One of the slats of the pantagraphs is connected at both ends by pins to the top table and the the ellipse curve plate. While one end of the other slat of the pantagraphs is connected by a pin to the base and a roller is attached at the other end and the top table is put on the roller. The center points of these two pairs of pantagraphs are linked by pins as illustrated in Fig. 2.

When the device is in motion, the top table moves vertically and along with the table, the angle $\theta$ in Fig. 2 varies. The ellipse curve plate pinned with the pantagraphs slides in the $x$-direction and the movable plates slides in the $y$-direction through the attached rollers. At that moment, the restoring force in the vertical direction is provided by the constant-force springs connected to the movable plate. 

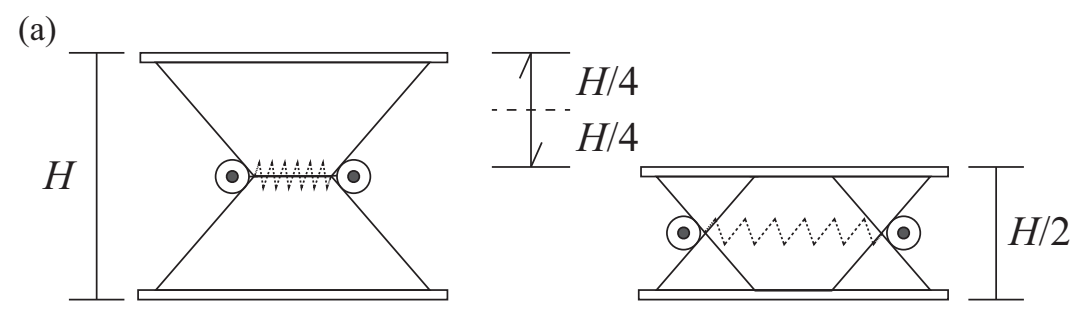

(b)
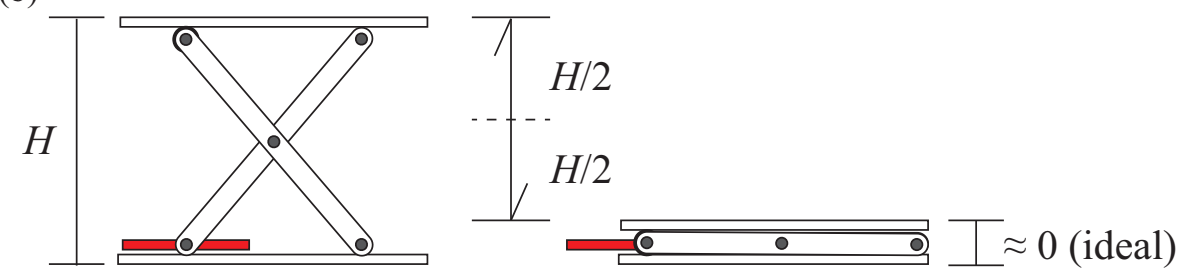

Figure 3. Advantages of the horizontal amplification mechanism

The advantages of the horizontally placed ellipse curve plate over the vertically placed trapezoidal one can be found in Fig. 3. Assume that the height of the device is $H$. Then the device with the vertically placed plate has an available stroke of $\pm H / 4$ as shown in Fig. 3 (a) while Fig. 3 (b) illustrates that the stroke produced by the horizontally placed plate is $\pm H / 2$. Also, the horizontal mechanism can make the height of the device ideally 0 when the top table reaches the bottom, while that value is $H / 2$ for the vertical mechanism due to the physical height of the vertically placed plates. Therefore, the horizontal mechanism have a lower risk of lack of stroke and realize a more compact isolator. These are advantages over other vertical isolators proposed in the literature including the authors' previous work [11, 12, 13, 14]. However, regardless of long stroke, impact absorption mechanism should be installed for practical use.

\subsection{Relationship between the restoring force and the shape of the horizontal amplification plate}

As stated before, for the vertical mechanism proposed in the literatures [13, 14], trapezoidal plates are used to amplify the restoring force of springs. Next, we justify that an ellipse curve plate is suited for the horizontal amplification mechanism to produce QZS for the vertical vibration isolator.

Consider an ellipse curve illustrated in Fig. 4 (a). The direction of compression of the device is defined as positive for the restoring force of the device and the relative displacement of the top table. Define that the $y$-direction component of the force acted on the point where the ellipse curve plate contacts with the roller attached to the movable plate as $n$ and the $x$-direction component as $t$. Define further that the length of the slat of the pantagraphs is $L$, the angle of the slat from the vertical direction is $\theta$, and the initial angle is $\theta_{0}$. It should be noted that $n$ equals to the restoring force of the springs, $t$ transfers to the $\mathrm{X}$-shaped pantagraphs, and $\theta$ is a function of $u$

As can be seen in Fig. 4 (b), the relationship between the restoring force of the device $f$ and horizontal force $t$ which is transmitted from the ellipse curve plate to the $\mathrm{X}$-shaped pantagraphs is expressed as

$$
f=\frac{t}{\tan \theta}
$$

Suppose that the ellipse curve is defined as $x=v(y)$ then the slope of the tangent becomes $d v / d y$. Thus the ratio of the vertical component to the horizontal one of the normal force acted on the plate is given by the slope of the tangent, i.e.,

$$
\frac{n}{t}=\frac{d v}{d y}
$$

From Eqs. (2) and (3), the amplification factor $\alpha$ would be

$$
\alpha=\frac{f}{n}=\frac{1}{\frac{d v}{d y} \tan \theta}
$$


(a)

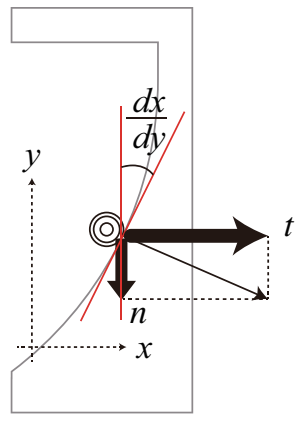

(b)

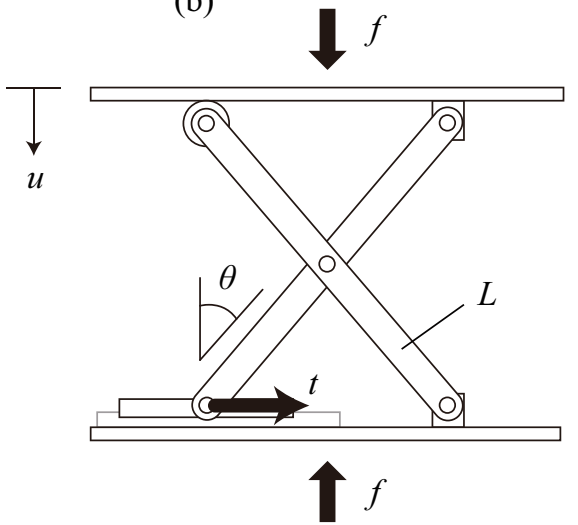

Figure 4. Forces acted on (a) Ellipse curve plate, (b) X-shaped pantagraphs.
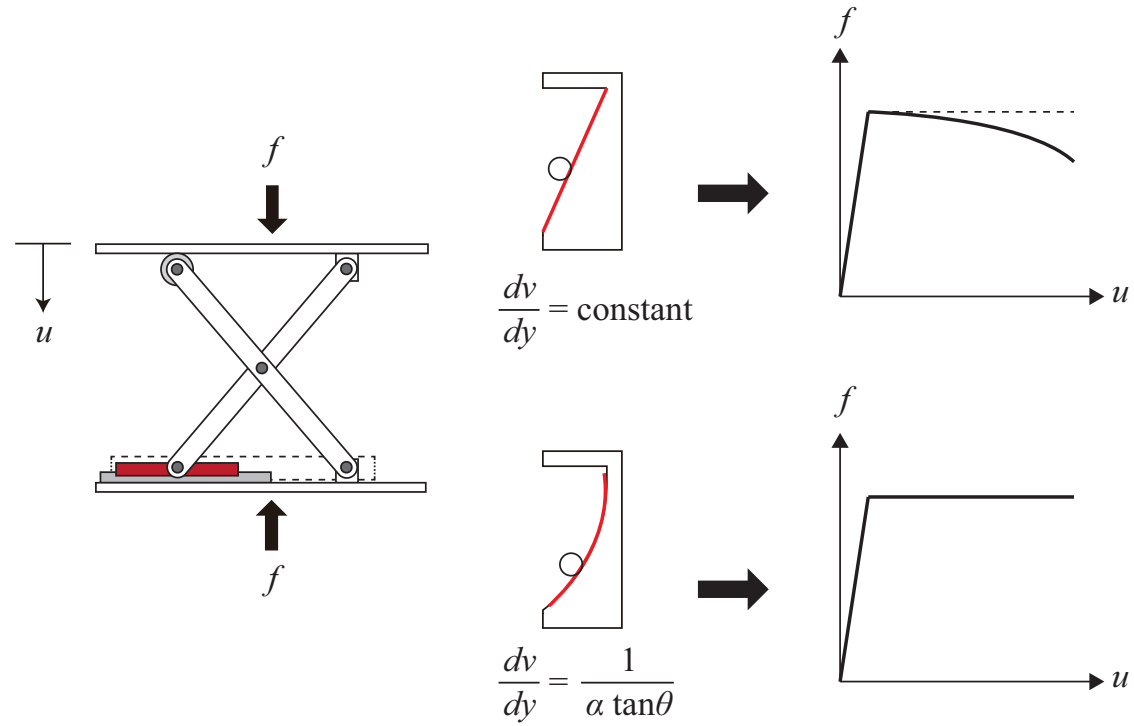

Figure 5. Properties of the restoring forces produced by horizontally placed plates

This equation implies that the amplification factor $\alpha$ depends on the slope of the tangent $d v / d y$ and the angle of pantagraphs $\theta$. Thus the amplification factor is determined by the shape of the horizontally placed plate. As illustrated in Fig. 5, when $d v / d y$ is constant, i.e., the shape of the horizontally placed plate is a linear, $\alpha$ becomes a function of $\tan \theta$. Thus $\alpha$ becomes a function of $u$ because $\theta$ is a function of $u$. Therefore the restoring force of the device depends on $u$. With this restoring force, it would be difficult to realize reliable isolators with long stroke. Also, without the horizontally placed plates, the same restoring force property could be produced by rotating the constant-force springs 90 degrees and connecting them directly to the $\mathrm{X}$-shaped pantagraphs as in $[15,16]$.

Next, consider constant $\alpha$ independent of $u$. In this case, the slope of the tangent is expressed as

$$
\frac{d v}{d y}=\frac{1}{\alpha \tan \theta}
$$

To verify that Eq. (5) represents the slope of the tangent of an ellipse curve, we show that $(x=v(y), y)$ satisfies the equation of the ellipse. The displacement of the top table $u$ is given by

$$
u=L\left(\cos \theta_{0}-\cos \theta\right)
$$


And the displacement of the ellipse curve plate corresponds to $v(y)$ because of the linear guides, thus the equation given by

$$
v(y)=L\left(\sin \theta-\sin \theta_{0}\right)
$$

holds. Because $\sin ^{2} \theta+\cos ^{2} \theta=1$, Eqs. (6) and (7) yield

$$
\left(\frac{u-L \cos \theta_{0}}{L}\right)^{2}+\left(\frac{v(y)+L \sin \theta_{0}}{L}\right)^{2}=1
$$

Also, from the conservation law of energy between the top table and the constant-force springs, we have

$$
f u=n y
$$

Thus Eq. (8) becomes

$$
\left(\frac{\frac{n}{f} y-L \cos \theta_{0}}{L}\right)^{2}+\left(\frac{v(y)+L \sin \theta_{0}}{L}\right)^{2}=1
$$

or

$$
\left(\frac{y-\alpha L \cos \theta_{0}}{\alpha L}\right)^{2}+\left(\frac{v(y)+L \sin \theta_{0}}{L}\right)^{2}=1
$$

Therefore $(x=v(y), y)$ satisfies the equation of the ellipse. Thus Eq. (5) is the slope of the tangent of the ellipse. From this derivation, once the initial angle $\theta_{0}$ and amplification factor $\alpha$ are determined, the shape of the ellipse curve $v(y)$ is determined. In this paper, $\pi / 4$ is used for $\theta_{0}$ and the semi axes of the ellipse becomes $L$ and $\alpha L$. This idea is applied to the variable ellipse curve mechanism according to the value of $\alpha$ for the adjustable vertical vibration introduced in the next section.

\section{ADJUSTABLE VERTICAL VIBRATION ISOLATOR WITH A VARIABLE ELLIPSE CURVE MECHANISM}

In this section, the horizontal amplification mechanism using the ellipse curve is realized by a variable mechanism which can change the semi axes according to the value of $\alpha$. Then an adjustable vertical vibration isolator is proposed and formulation of the restoring force of the proposed device follows.

\subsection{Variable ellipse curve mechanism}

As derived in the previous section, the shape of the horizontally placed plate for the QZS vibration isolators is ellipse curve whose semi axes have length of $L$ and $\alpha L$. This result tells us that the amplification factor $\alpha$ (i.e., the restoring force of the device is $f=\alpha n$ ) can be variable if the length $\alpha L$ is adjustable. Here we propose a mechanism which can change the length $\alpha L$ continuously by applying the idea of ellipsographs.

Fig. 6 depicts the trajectory of an ellipsograph, which consists of a straight bar and two linearly movable parts (points A and B) in a cross slider. As can be seen in the figure, an ellipse curve can be drawn by sliding these two parts crosswise. To install this mechanism on the isolator, because constant-force springs stretch only in one direction, the end point of the straight bar of the ellipsograph (point $\mathrm{C}$ ) is connected to the spring by a pin and the cross slider part is designed to be movable in the direction perpendicular to the stretch direction of the constant-force springs as shown in Fig. 7 (a). Fig. 7 (b) shows the decomposed force at the pin (Point C) connected to the spring. By comparing with Fig. 4 (a), this mechanism can convert the same force from the springs as the ellipse curve plate introduced in the previous section. Thus the equations derived for the ellipse curve plate still hold. Fig. 7 (c) shows that the relative motion of the movable cross slider as seen from Point $\mathrm{C}$ draws as ellipse trajectory as well.

Next, the relationship between the trajectory of ellipse and the amplification factor $\alpha$ is investigated. Fig. 8 shows trajectories of ellipses as the values of $\alpha$ increases from 0 . As can 


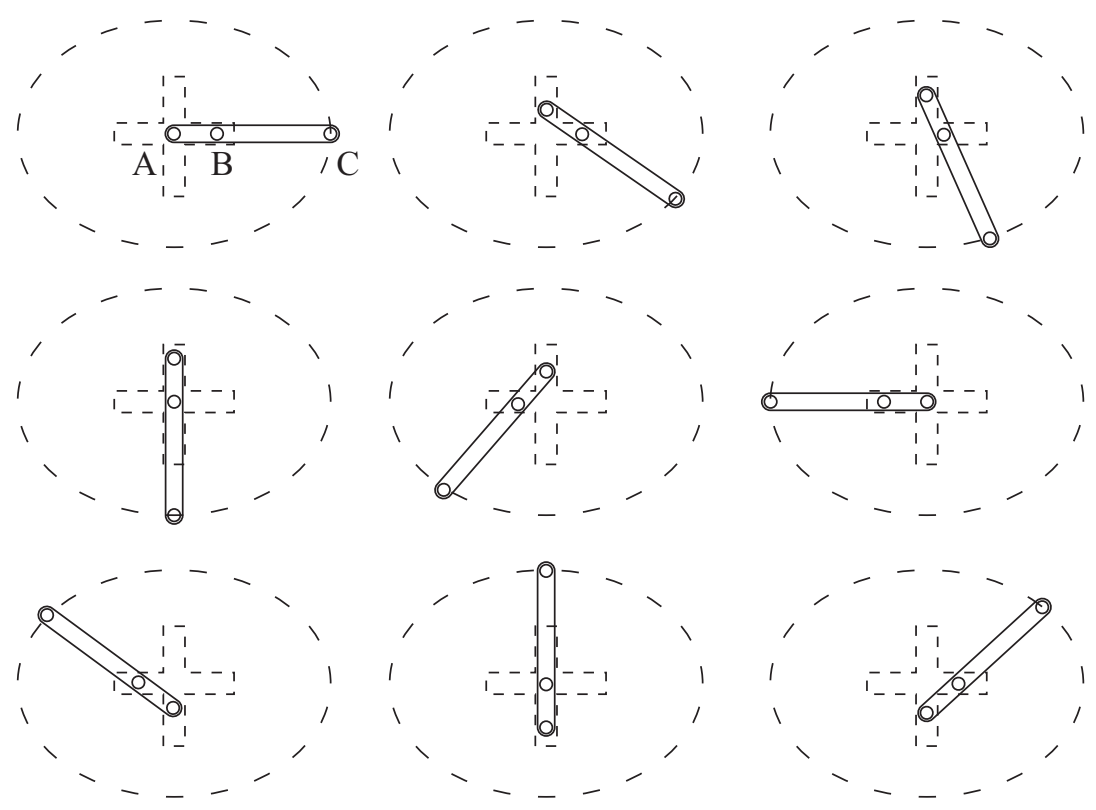

Figure 6. Trajectory of an ellipsograph

(a)

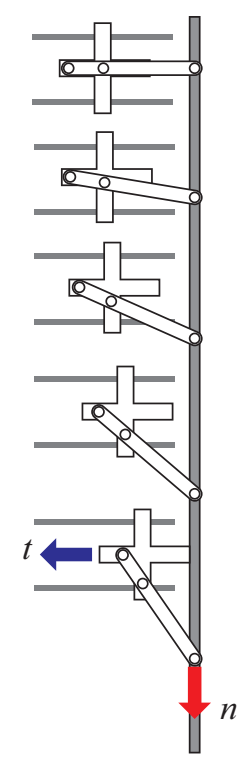

(b)

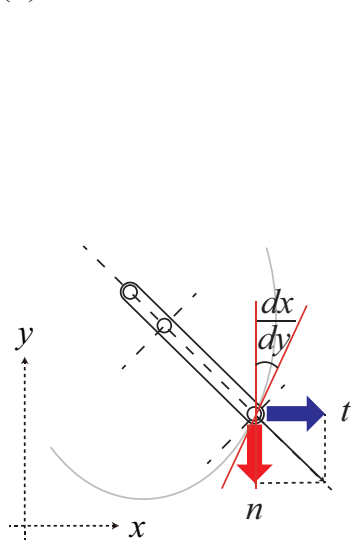

(c)
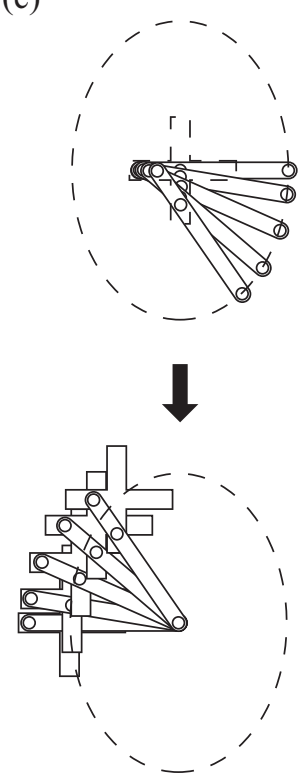

Figure 7. Constrained ellipsograph with a movable cross slider. (a) Schematic illustration, (b) Acted force, (c) Comparisons of the trajectories

be observed in the figure, the semi-minor and semi-major axes are switched when $\alpha$ reaches 1 . The trajectories and behaviors of the ellipsograph with the movable cross slider for the cases of $0<\alpha \leq 1$ and $1 \leq \alpha$ are shown in Fig. 8 (b) and (c). The figure shows by red dotted boxes that the ranges in which points $\mathrm{A}$ and $\mathrm{B}$ moves differ for the cases of $0<\alpha \leq 1$ and $1 \leq \alpha$. Also, the length of $\mathrm{BC}$ and $\mathrm{AC}$ correspond to $\alpha L$ for the cases of $0<\alpha \leq 1$ and $1 \leq \alpha$, respectively. Therefore it is not easy to design a prototype which can realize any $\alpha$. Thus in this paper, a prototype for the case of $0<\alpha \leq 1$, i.e., $\mathrm{AC}=L$ and $\mathrm{BC}=\alpha L$, as shown in Fig. 8 (b) is examined. 
(a)

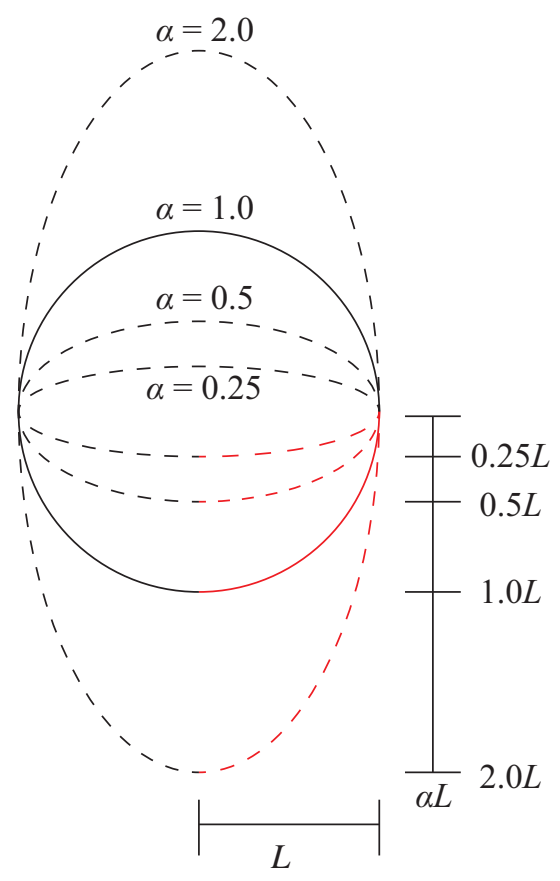

(b)
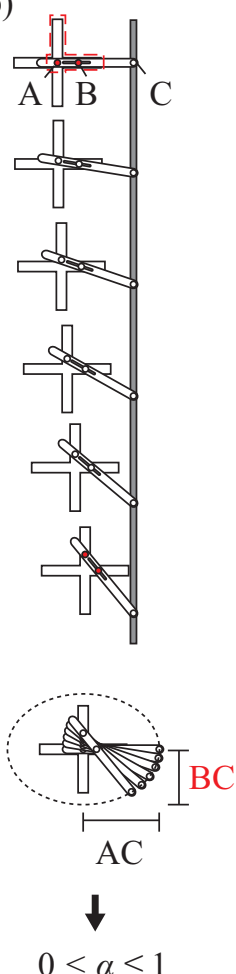

(c)
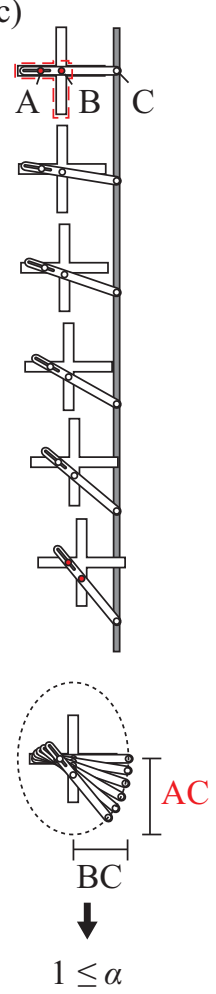

Figure 8. Relationship between eclipse curves and amplification factors. (a) Eclipse curve for each $\alpha$, (b) $0<\alpha \leq 1$, (c) $1 \leq \alpha$

\subsection{Design of the adjustable vertical vibration isolator}

Fig. 9 depicts an axonometric drawing of the proposed vertical vibration isolator, and Fig. 10 shows the plan and elevation of the device with different height states. As can be seen in Fig. 9 (b), the proposed device can be broken down into three parts, i.e., upper, middle, and lower parts. The upper part is a top table on which target objects are placed. The middle part consists of two pairs of Xshaped pantagraphs which transfers the restoring force between the upper and lower parts. These two pairs installed on the front and back sides of the device are connected by plates. By adjusting the stiffness and flexibility of this part, rocking vibration can be minimized. The lower part plays a key role to realize the restoring force using the variable ellipse curve mechanism. The restoring force produced by the constant-force springs are transferred and scaled via the variable ellipse curve mechanism to a sliding bar, then transferred to the X-shaped pantagraphs which are connected to the sliding bar.

The detail of the variable ellipse curve mechanism is explained. The red-colored part corresponds to the straight bar of the ellipsograph. The variable ellipse curve mechanism has three pinned joints, $\mathrm{A}, \mathrm{B}$, and $\mathrm{C}$ which correspond to the points, $\mathrm{A}, \mathrm{B}$, and $\mathrm{C}$ in Fig. 8 (c). The pin $\mathrm{A}$ is connected to the linear guide on the sliding bar and the pin A can move in a straight line on the sliding bar in the $y$-direction in Fig. 10. The sliding bar moves on the two linear guides installed on the base of the device in the $x$-direction. Thus the pin A can move in the directions of both $x$ and $y$ axes. The pin B is hooked to the liner guide on the center line of the base plate and the pin B slides in the $x$-direction along with the sliding bar. The pin $\mathrm{C}$ is linked to the movable plate which is connected to the constant-force springs and the plate is movable in the $y$-direction.

When the device is in motion, the variable ellipse curve mechanism slides on the linear guides and rotates accordingly. The part in the dotted box in Fig. 10 works as the cross slider part of ellipsographs. Therefore the ellipsograph mechanism is realized by the proposed variable ellipse curve mechanism. The device in this paper has two vertically overlapped variable ellipse curve 
(a)

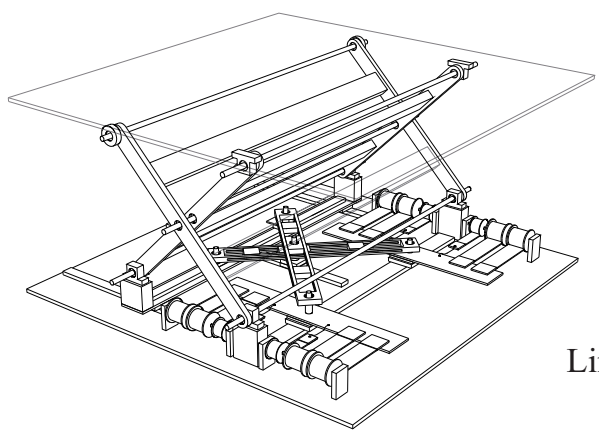

(b)

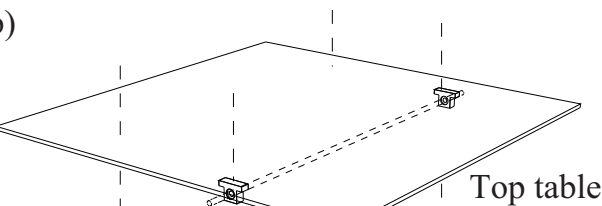

Variable mechanism

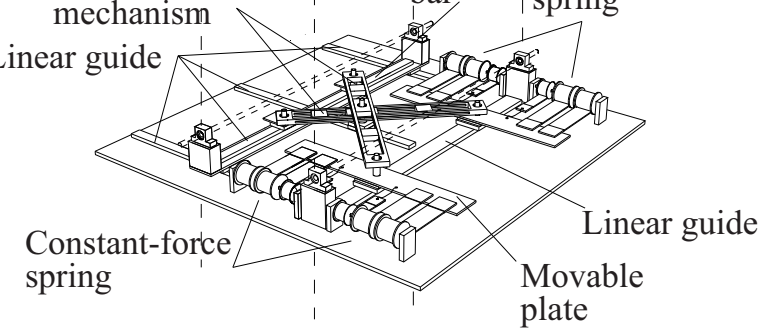

Figure 9. Axonometric drawing of the proposed device. (a) Overall, (b) Divided

(a)
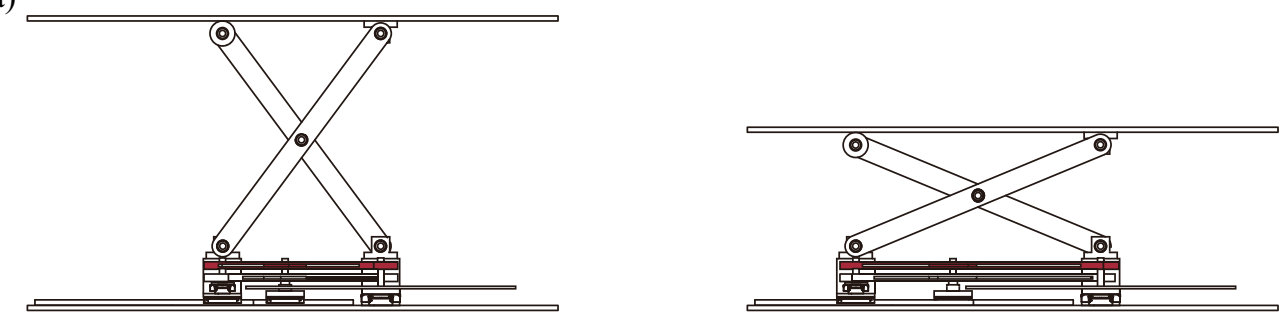

(b)

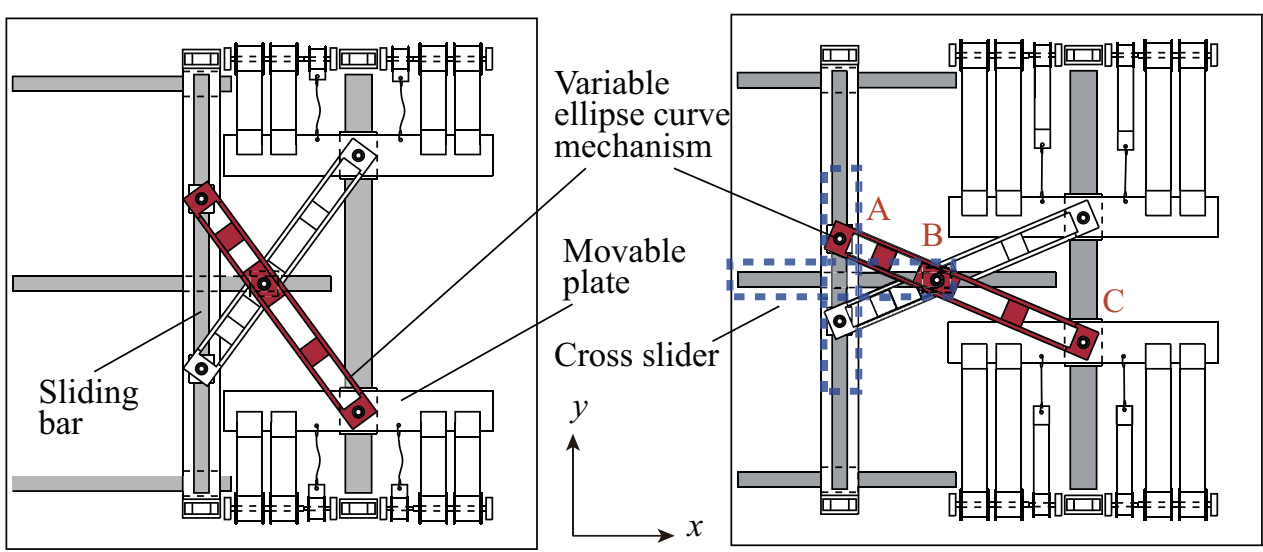

Figure 10. Schematic illustration of the proposed device. (a) Elevation, (b) Plan.

mechanisms as shown in Fig. 10. These two mechanisms makes the device symmetry and prevent from rocking. In addition, the weight limit of the target object is increased. 
To change the ellipse curve for the amplification factor satisfying $0<\alpha \leq 1$, the location of the pin $\mathrm{B}$ is designed to be adjustable between the points $\mathrm{A}$ and $\mathrm{C}$. The length of $\mathrm{BC}$ corresponds to the semi-minor axis BC in Fig. 8. Let $r$ be the length of BC, then the relationship with $\alpha$ is expressed, from Fig. 8, as

$$
r=\alpha L
$$

The amplification factor $\alpha$ is decided by the weight of the target object. Thus the appropriate length of BC can be calculated by Eq. (12).

Finally, as can be seen in Fig. 9, any dampers are not installed in the proposed device. This is because as discussed in the next section, considerable friction force is measured and enough damping is already obtained to absorb input energy. Therefore installing additional dampers might create risks of losing the self-centering capacity and increasing response acceleration. Also due to the nonlinear restoring force property, resonance is not a serious issue in the proposed mechanism. Thus the prototype designed for this research does not need to have any dampers. However, depending on circumstances, adding dampers is worth considering to improve the vibration reduction capability of the proposed mechanism. In this case, friction dampers or shock absorbers can be accommodated horizontally in parallel with the constant-force springs, which maintains the height advantage.

\subsection{Flag-shaped restoring force}

The proposed device is designed to produce flag-shaped restoring force. In this subsection, the mechanism and its properties are described. Fig. 11 illustrates the states of the constant-force springs for the upper limit, static equilibrium, and lower limit positions. To produce flag-shaped restoring force, springs with large and small constant-forces are used. The large and small ones are called constant-force springs 1 and 2, respectively in this paper. The constant-force springs 2 are connected through wires as shown in Fig. 11. By doing this, the springs 2 do not work until the springs 1 deform to some extent, then flag-shaped restoring force is realized. Hereafter, the stretch of the constant-force springs 1 is defined as $w$ and the tensile direction is defined as positive. The relative displacement of the top table are defined as $u$ and its positive direction is downward. Fig. 11 (a) depicts the upper limit position where the top table is at the highest position. This is determined by the location of a stopper for the sliding bar. At this position, $u$ and $w$ are set to 0 . The static equilibrium position when the target object is placed on the top table is shown in Fig. 11 (b). At the equilibrium position, $u$ and $w$ are defined as $u_{\mathrm{se}}$ and $w_{\mathrm{se}}$, and $m$ is the mass of the target object including the top table and $g$ is gravitational acceleration. Lastly, Fig. 11 (c) shows the state where the top table is at the bottom, and $u$ and $w$ at this state are defined as $u_{\max }$ and $w_{\max }$.

Now we investigate how $n$ changes as $w$ increases from 0 to $w_{\max }$. When $w=0$, the restoring force $n$ changes from 0 to $N_{1}$, which is the total force of the constant-force springs 1 . When $0<w<w_{\max }$, the springs 1 contribute $N_{1}$ to the restoring force. While the springs 2 which are connected through the wires apply force ranging from 0 to $N_{2}$, which is the total force of the constant-force springs 2, as the part of the restoring force. If $w$ is relatively small, the wires sagged and the springs 2 are not contributed to the restoring force at all and we have $n=N_{1}$. While the top table moves downward, the wires starts to perform tensile force and contribute $N_{2}$ load to the restoring force. At that moment, $n=N_{1}+N_{2}$.

Fig. 12 shows the relationships between $f$ and $u$, and $n$ and $w$. Henceforth another shifted coordinate systems in terms of the static equilibrium position defined as

$$
\Delta f=f-m g, \quad \Delta u=u-u_{s e}, \quad \Delta n=n-\frac{m g}{\alpha}, \quad \Delta w=w-w_{s e}
$$

are used. These coordinate systems are shown in Fig. 12 as well. Also, the relationship between $u$ and $w$ given by

$$
u=\frac{n}{f} w=\frac{1}{\alpha} w
$$

holds from the conservation law of energy as Eq. (9), 
(a)

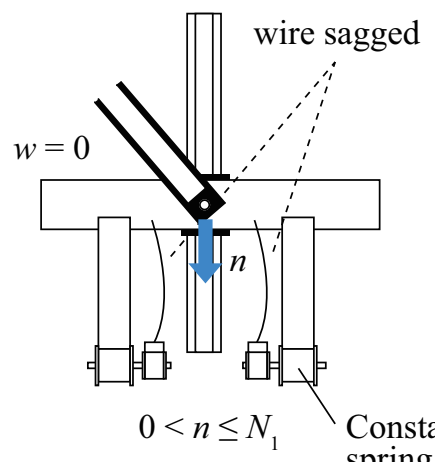

(b)

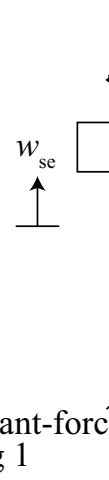

(c)

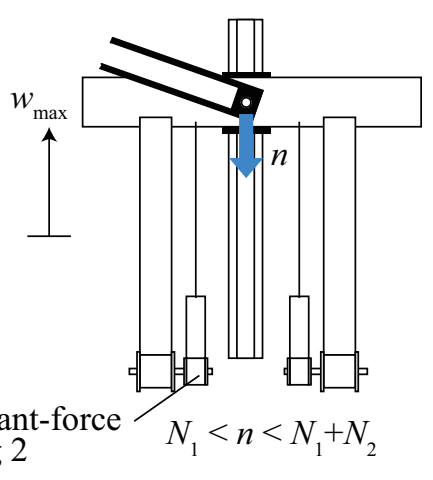

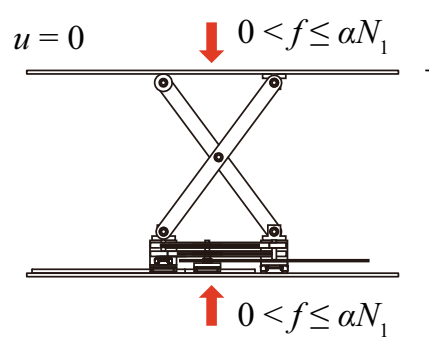
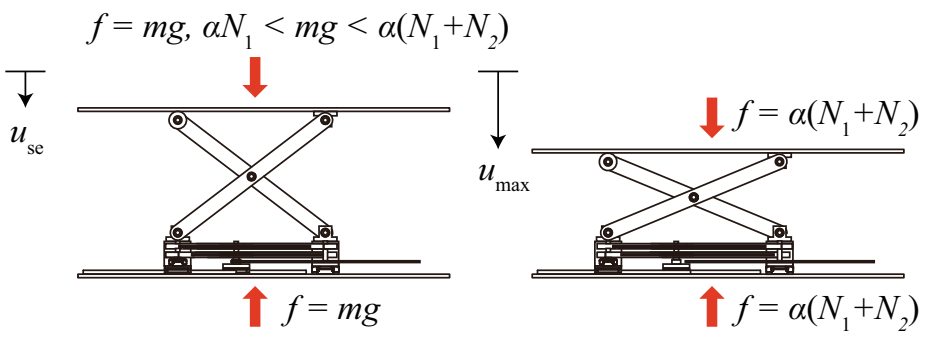

Figure 11. Mechanism for producing nonlinear restoring force. (a) Upper limit position, (b) Static equilibrium position, (c) Lower limit position.

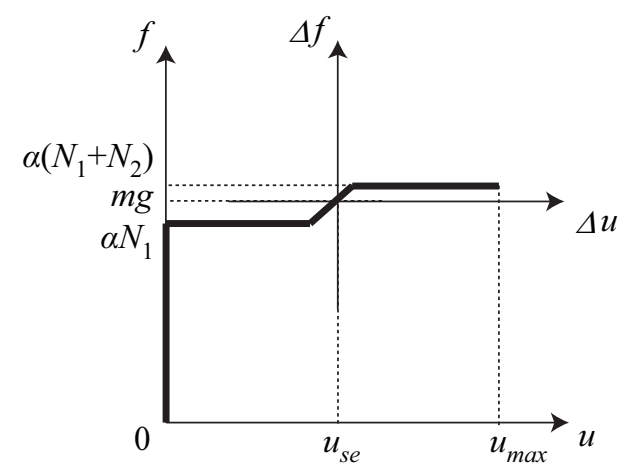

(a)

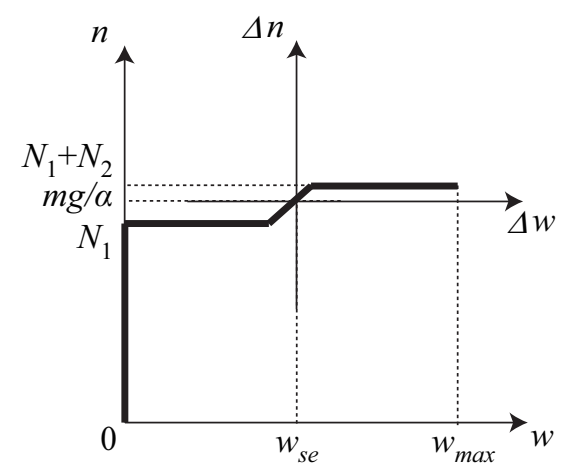

(b)

Figure 12. Relationships between (a) $f$ and $u$, (b) $n$ and $w$.

The numerical models for the restoring force and the friction are depicted in Fig. 13. Fig. 13 (a) shows the numerical model for the relationship between the shifted restoring force $\Delta n$ and the shifted relative displacement $\Delta w$ where no friction is considered. Practically, constant-force springs have certain stiffness $k$ until the tension force becomes constant. Thus the restoring force can be modeled as

$$
\Delta n=n-\frac{m g}{\alpha}= \begin{cases}N_{1}-m g / \alpha & \text { if }-w_{s e}<\Delta w<\Delta w_{L} \\ k \Delta w & \text { if } \Delta w_{L} \leq \Delta w \leq \Delta w_{U} \\ N_{1}+N_{2}-m g / \alpha & \text { if } \Delta w_{U}<\Delta w<w_{\max }-w_{s e}\end{cases}
$$

where

$$
\Delta w_{U}=\frac{N_{1}+N_{2}-m g / \alpha}{k}, \quad \Delta w_{L}=\frac{N_{1}-m g / \alpha}{k}
$$


(a)

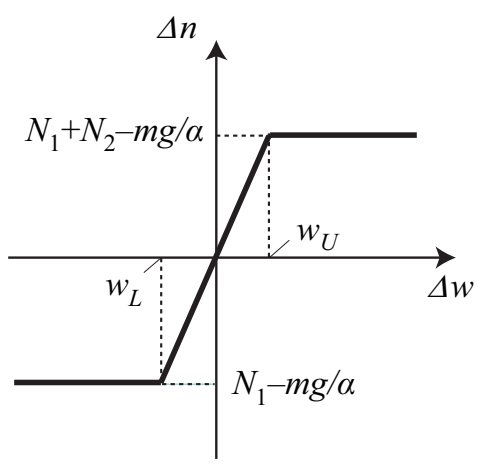

(b)

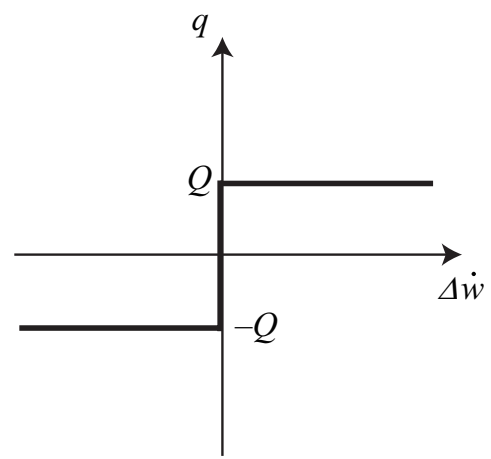

Figure 13. Numerical models for (a) Restoring force, (b) Friction

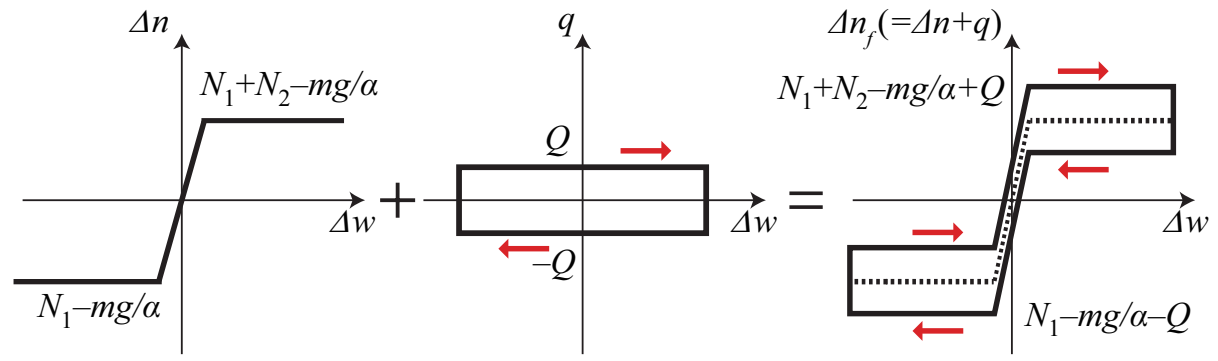

Figure 14. Flag-shaped restoring force

We assume that the device have unavoidable Coulomb's friction $q$ modeled as

$$
q= \begin{cases}-Q & \text { if } \Delta \dot{w}<0 \\ 0 & \text { if } \Delta \dot{w}=0 \\ Q & \text { if } \Delta \dot{w}>0\end{cases}
$$

where $Q>0$ and the overdot indicates the differentiation with respect to time. This is illustrated in Fig. 13 (b).

Summing up these forces, the restoring force including the friction can be expressed as

$$
\Delta n_{f}(\Delta w, \Delta \dot{w})=\Delta n(\Delta w)+q(\Delta \dot{w})
$$

and this is shown in Fig. 14 graphically. From the look of the shape, this is called flag-shape restoring force. Let $\Delta f_{f}$ be the restoring force of the device including the friction force. Then the relationship between $\Delta f_{f}$ and $\Delta n_{f}$ is given by

$$
\Delta f_{f}=\alpha \Delta n_{f}
$$

thus $\Delta f_{f}$ is obtained as

$$
\Delta f_{f}(\Delta u, \Delta \dot{u})= \begin{cases}\alpha\left(N_{1}+q(\alpha \Delta \dot{u})\right)-m g & \text { if }-u_{s e}<\Delta u<w_{L} / \alpha \\ \alpha^{2} k \Delta u+\alpha q(\alpha \Delta \dot{u}) & \text { if } w_{L} / \alpha \leq \Delta u \leq w_{U} / \alpha \\ \alpha\left(N_{1}+N_{2}+q(\alpha \Delta \dot{u})\right)-m g & \text { if } w_{U} / \alpha<\Delta u<u_{\text {max }}-u_{s e}\end{cases}
$$

It should be noted that by adjusting $\alpha$ within the range of

$$
\alpha N_{1}<m g<\alpha\left(N_{1}+N_{2}\right) \quad \text { or } \quad \frac{m g}{N_{1}+N_{2}}<\alpha<\frac{m g}{N_{1}}
$$

even when $m g$ changes, the proposed device can be self-centered, i.e., the upper table returns to the static equilibrium position after the external disturbance stops. 
The properties of the vertical vibration isolator with the variable ellipse curve mechanism and its flag-shaped restoring force are summarized as follows:

1. The capacity of the isolator can be adjustable continuously by the simple variable ellipse curve mechanism.

2. The flat part of the restoring force curve limits the response acceleration within a specified tolerance regardless of the amplitude and frequency of input motions.

3. The flag-shaped restoring force provides the isolator with the self-centering capability. This is because static equilibrium can be achieved only at the origin of the restoring force curve. The self-centering capability prevents the device from accumulating deformation in one direction in vibration, which may be seen when friction damping is applied [17, 18, 19].

4. Resonance can be avoided by flag-shaped restoring force. The proposed isolator responses linearly in the neighborhood of the static equilibrium point. In this linear range, the isolator has a potential of resonance. Nevertheless, this does not lead to serious problems because the displacement amplitude of interest in this paper is well beyond the linear range. Even if resonance takes place in the linear range, the equivalent natural frequency (computed either by the secant stiffness or by the tangent stiffness) decreases significantly as the displacement amplitude increases beyond the linear range. The change of the equivalent natural frequency with respect to the displacement amplitude avoids resonance.

The second property mentioned above can be explained in the following way. The equation of motion of the target object placed on the proposed device would be

$$
m\left(\Delta \ddot{u}+\ddot{u}_{g}\right)+c \Delta \dot{u}+\Delta f_{f}(\Delta u, \Delta \dot{u})=0
$$

where $\ddot{u}_{g}$ is ground acceleration. Suppose that the damping coefficient $c$ is small enough, then from Eqs. (17), (20), and (22), the maximum value of the response absolute acceleration $\left|\Delta \ddot{u}+\ddot{u}_{g}\right|$ would be constrained by

$$
\left|\Delta \ddot{u}+\ddot{u}_{g}\right| \leq h(\alpha)
$$

where

$$
h(\alpha)=\max \left(g-\frac{\alpha}{m}\left(N_{1}-Q\right), \frac{\alpha}{m}\left(N_{1}+N_{2}+Q\right)-g\right)
$$

Eqs. (23) and (24) imply that the maximum response absolute acceleration can be determined by the value of $\alpha / m$. Therefore for various $m$, the response absolute acceleration can be ideally restrained less than the particular value by adjusting $\alpha$.

Consider the range of $h(\alpha)$ for $\alpha$ satisfying Eq. (21). Both elements in Eq. (24) are linear functions of $\alpha$, thus $h(\alpha)$ is a piecewise linear function of $\alpha$. Hence the range of $h(\alpha)$ is obtained from Fig. 15 , as

$$
\frac{N_{2} / 2+Q}{N_{1}+N_{2} / 2} g \leq h(\alpha)<\frac{N_{2}+Q}{N_{1}} g
$$

From this derivation, we can conclude that if $c$ is ignorable, the response absolute acceleration can be constrained by $\left(N_{2}+Q\right) g / N_{1}$ regardless of $m$.

\section{EXPERIMENTAL VERIFICATION}

The effectiveness of the vertical vibration isolator with the proposed variable ellipse curve mechanism is verified through shaking table tests in this section. A prototype of the device is made and its parameters are determined by quasi-static loading tests. Then a variety of sine waves and scaled earthquake records are input to the prototype in the only vertical direction because the device is assumed to be used in combination with horizontal isolators.

\subsection{Prototype of the proposed device}

Fig. 16 shows photographs of the prototype of the proposed device. The length of the X-shaped pantagraphs $L$ is $424 \mathrm{~mm}$ and the initial angle $\theta_{0}$ is $\pi / 4$, thus the hight of the device is $300 \mathrm{~mm}$ at a 


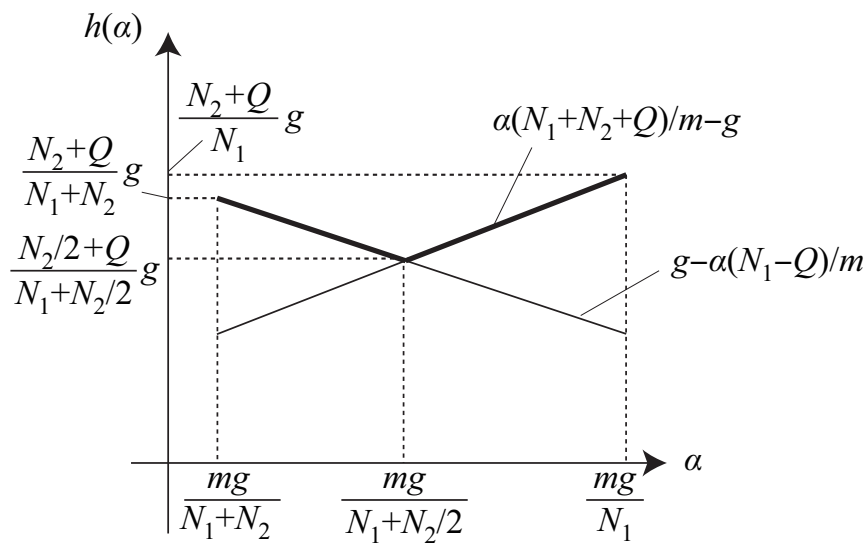

Figure 15. Relationship between $h(\alpha)$ and $\alpha$
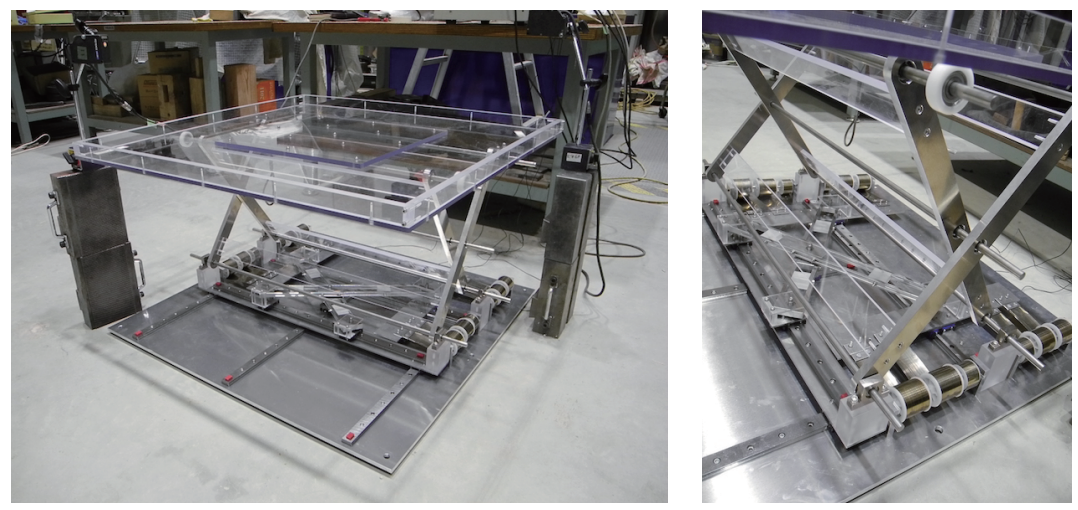

Figure 16. Photographs of the prototype of the proposed device

Table I. Reference parameters for the restoring force

\begin{tabular}{ccccc}
\hline$r(\mathrm{~mm})$ & $\alpha$ & $N_{1(\alpha)}(\mathrm{N})$ & $N_{2(\alpha)}(\mathrm{N})$ & $Q_{(\alpha)}(\mathrm{N})$ \\
\hline 170 & 0.40 & 156.8 & 26.7 & 14.21 \\
255 & 0.60 & 235.2 & 40.1 & 21.31 \\
339 & 0.80 & 313.6 & 53.4 & 28.41 \\
\hline
\end{tabular}

static equilibrium position. To minimize overturning effect acting on the isolated object on the table, the stiffness and flexibility of the $\mathrm{X}$-shaped panagraphs are fine-tuned by adjusting the screws on the prototype used in this study. The moderate state is decided after conducting preliminary shaking table tests by trial and error. However further examination is needed for the device to provide an explicit tuning method. The minimum and maximum heights of the device are $120 \mathrm{~mm}$ and 480 $\mathrm{mm}$, respectively, i.e., the device ensures $\pm 180 \mathrm{~mm}$ stroke from the equilibrium position.

The restoring forces of the constant-force springs 1 and 2 used for the device are $N_{1}=392.0 \mathrm{~N}$ (49.0 N each $\times 8$ springs) and $N_{2}=68.8 \mathrm{~N}(16.7 \mathrm{~N}$ each $\times 4$ springs). The standard friction force $Q$, which is determined from the quasi-static test described next, is obtained as $35.52 \mathrm{~N}$. For each amplification factor $\alpha$, the parameter values are calculated by

$$
N_{1(\alpha)}=\alpha N_{1}, \quad N_{2(\alpha)}=\alpha N_{2}, Q_{(\alpha)}=\alpha Q
$$

These values are summarized in Table I. 
(a)

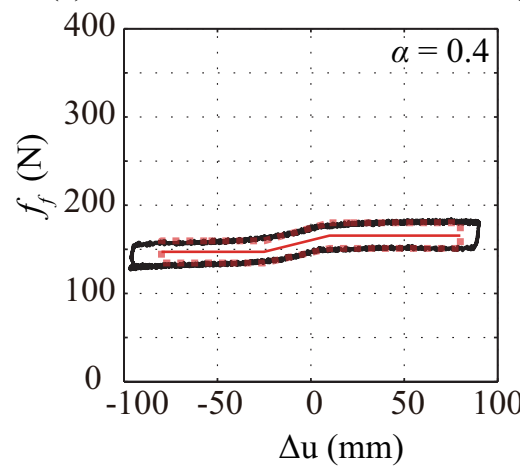

(b)

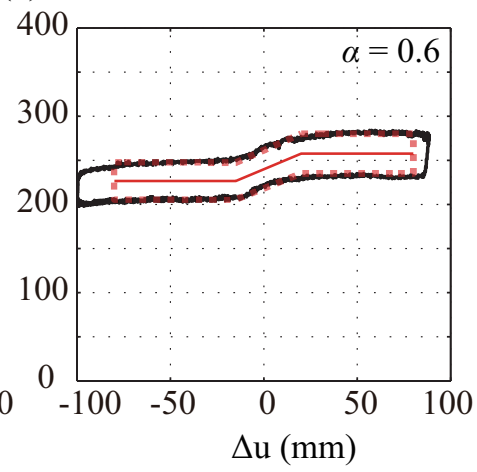

(c)

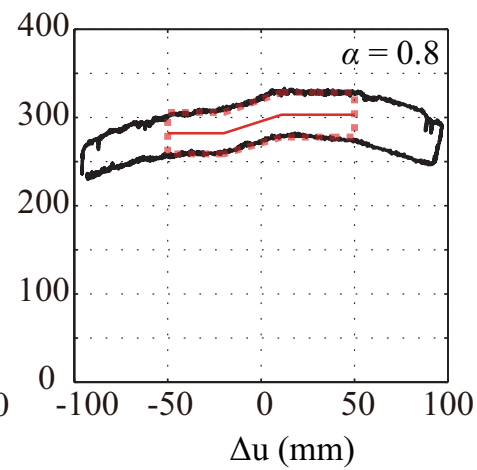

Figure 17. Restoring forces obtained from quasi-static tests (black) and their mathematical model (red)

Table II. Experimental parameters for the restoring force

\begin{tabular}{ccccc}
\hline$r(\mathrm{~mm})$ & $\alpha$ & $N_{1(\alpha)}(\mathrm{N})$ & $N_{2(\alpha)}(\mathrm{N})$ & $Q_{(\alpha)}(\mathrm{N})$ \\
\hline 170 & 0.40 & 146.1 & 19.4 & 13.83 \\
255 & 0.60 & 226.5 & 31.0 & 21.63 \\
339 & 0.80 & 282.2 & 20.9 & 24.4 \\
\hline
\end{tabular}

\subsection{Quasi-static loading tests}

To obtain the parameter values for the mathematical model of the prototype, quasi-static loading tests are conducted. Vertical uniaxial quasi-static force is applied on the upper table manually. The tests are implemented for three amplification factors, 0.4, 0.6, and 0.8. These amplification factors correspond to $170 \mathrm{~mm}, 255 \mathrm{~mm}$, and $339 \mathrm{~mm}$ of the semi-minor axis $r$ (i.e., the length of $\mathrm{BC}$ in Fig. 10). The displacement of the top table is defined as the average of two displacement sensors measuring two opposing points on the table. The restoring force is measured by summing up three load cells installed between the ground and the base of the device.

Fig. 17 shows the friction-included restoring force $f_{f}$ obtained from the tests and the calculated parameters are given in Table II. As can be seen in Fig. 17, the device does not provide complete self-centering restoring force for all cases due to the large friction force. However, according to [20], in which self-centering capacities for various kinds of restoring force are reported, the condition to ensure self-centering capacity for the proposed device to most earthquake disturbances can be derived as

$$
\frac{N_{2}}{2}+Q>2\left|\frac{N_{2}}{2}-Q\right|
$$

under the assumption that $N_{1}+N_{2} / 2=m g / \alpha$. We can confirm from Table II that the prototype satisfies Eq. (27), thus sufficient self-centering capacity can be expected from the device.

Fig. 18 compares the reference and experimental values for $N_{1(\alpha)}$ and $N_{1(\alpha)}+N_{2(\alpha)}$. As can be seen, the experimental values become smaller than the reference values. However, these values are matched well when $\alpha$ is small, while the differences becomes larger as $\alpha$ increases. One of the possible causes is the design flaw of the prototype for the case of large $\alpha$. To reduce the discrepancy, further improvement of the design is required.

In Fig. 19, the relationship between experimental values of $N_{1(\alpha)}$ and $Q_{(\alpha)}$ is plotted by x. The linear approximation, which is shown by red line in Fig. 19, is expressed by

$$
Q_{(\alpha)}=\beta N_{1(\alpha)}
$$


(a)

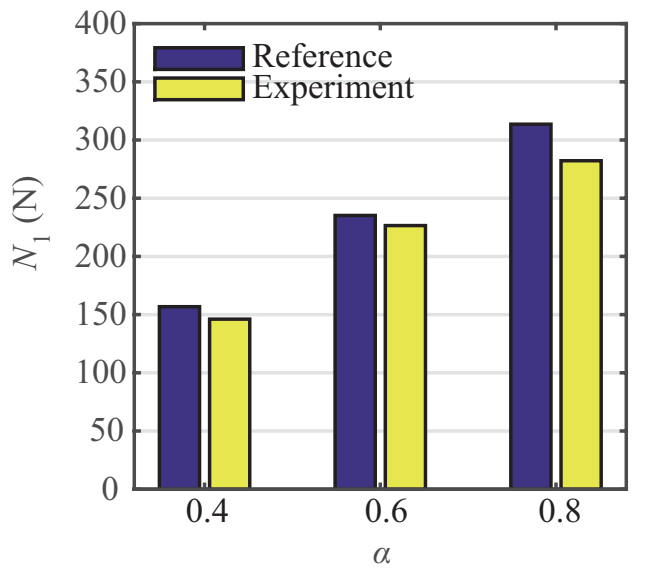

(b)

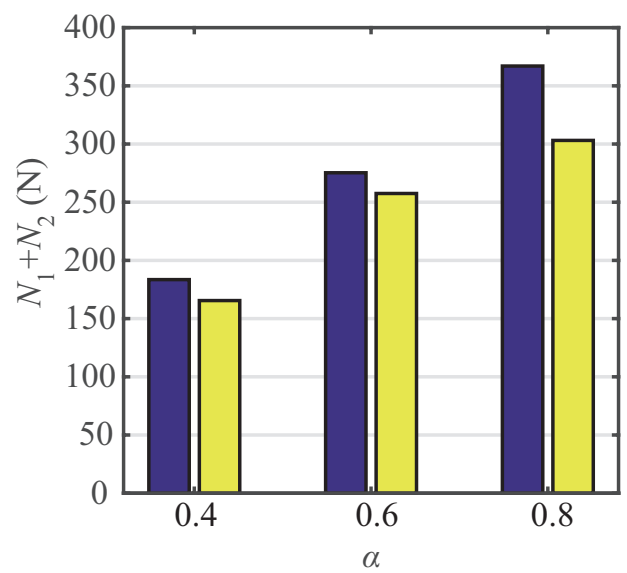

Figure 18. Comparisons of reference and experimental values for the device. (a) $N_{1}$, (b) $N_{1}+N_{2}$

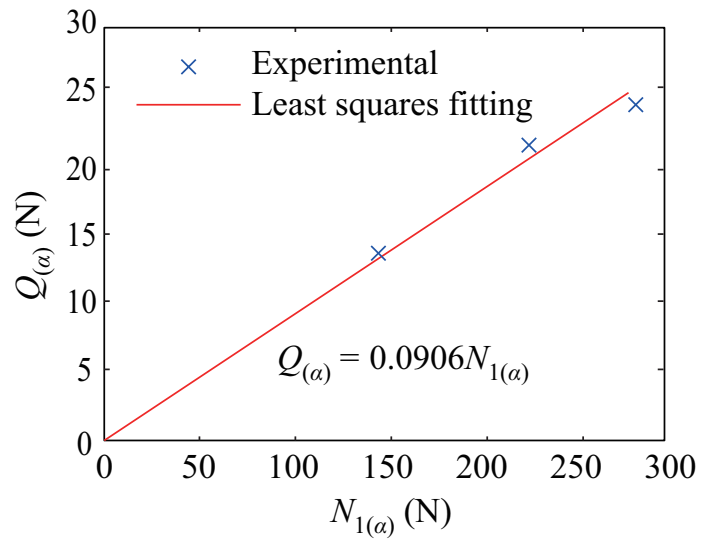

Figure 19. Relationship between $N_{1(\alpha)}$ and $Q_{(\alpha)}$

where $\beta$ is determined as 0.0906 by the method of least squares fitting. $Q_{(\alpha)}$ in Table I is defined as the product of $\beta$ and $N_{1(\alpha)}$. Then the value of the standard friction $Q$ used in Eq. (26) is determined. This figure shows that the friction force increases in proportion to the weight of the target object.

\subsection{Shaking table tests}

To verify the validity of the proposed device for various weights, shaking table tests are carried out. The experimental setup is illustrated in Fig. 20. Input ground motions have only vertical or up-down (UD) component. The amplification factor $\alpha$ is adjusted to 0.4, 0.6, and 0.8. For these three cases, $15.82 \mathrm{~kg}, 24.85 \mathrm{~kg}$, and $29.87 \mathrm{~kg}$ masses of the target object are employed. These masses include the upper table $(12.85 \mathrm{~kg})$. Acceleration sensors are installed on the upper table of the vibration isolator to measure response acceleration (RA), and on the shaking table for ground acceleration (GA). Non-contact laser displacement sensors are fixed to a measurement frame to measure response absolute displacement (RAD) and ground displacement (GD). Response relative displacement (RRD) is obtained by subtracting GD from RAD.

Table III summarizes the input excitations. $3 \mathrm{~Hz}$ and $5 \mathrm{~Hz}$ size waves and the UD component of the earthquake ground motion recorded at K-NET Ojiya station in Japan during the 2004 Mid Niigata Prefecture earthquake. These excitations are normalized so that the peak ground accelerations (PGAs) become $0.50 \mathrm{~g}$ and $1.00 \mathrm{~g}$, whereas the PGA of the original Ojiya record is $0.83 \mathrm{~g}$. 


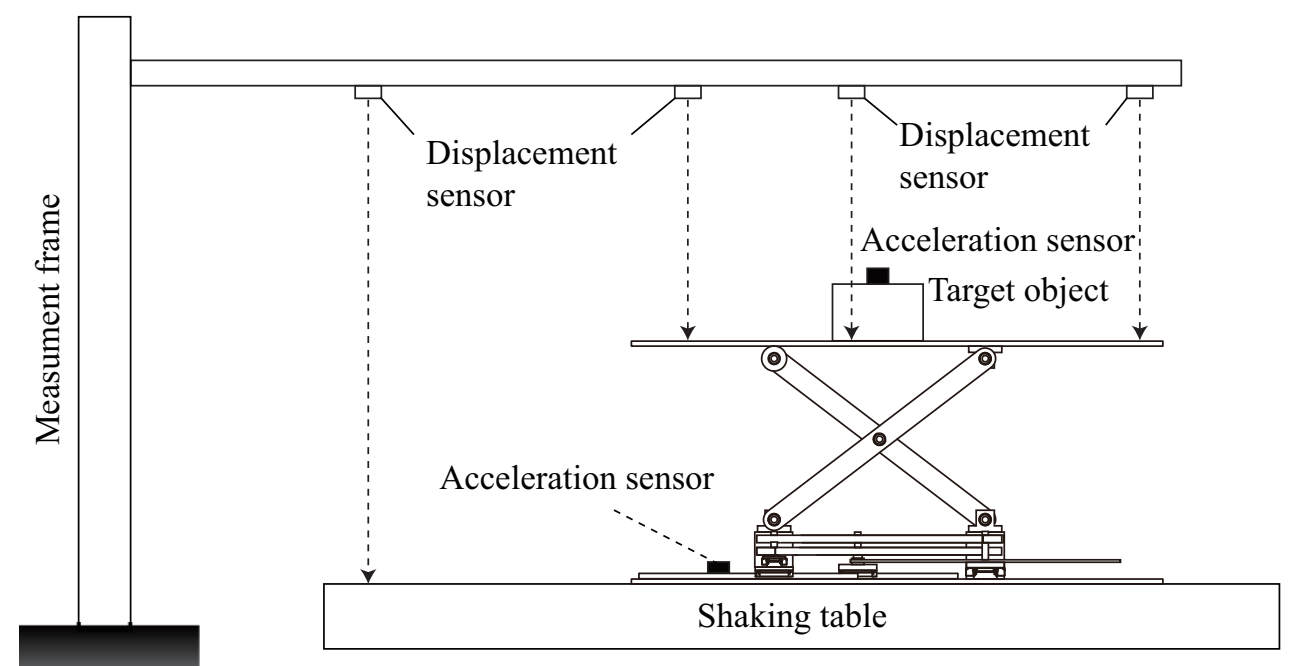

Ground

Figure 20. Experimental setup for shaking table tests

Table III. List of input excitations

\begin{tabular}{ccc}
\hline & Input & Target PGA $(\mathrm{g})$ \\
\hline S3-1 & 3Hz sine wave & 0.50 \\
S3-2 & 3Hz sine wave & 1.00 \\
S5-1 & 5Hz sine wave & 0.50 \\
S5-2 & 5Hz sine wave & 1.00 \\
O-1 & K-NET Ojiya UD & 0.50 \\
O-2 & K-NET Ojiya UD & 1.00 \\
\hline
\end{tabular}

Table IV. Comparisons between PGA and PRA

\begin{tabular}{ccccccc}
\hline$\alpha$ & \multicolumn{2}{c}{0.4} & \multicolumn{2}{c}{0.6} & \multicolumn{2}{c}{0.8} \\
\hline Input & PGA (g) & PRA (g) & PGA (g) & PRA (g) & PGA (g) & PRA (g) \\
\hline S3-1 & 0.552 & 0.240 & 0.563 & 0.205 & 0.642 & 0.243 \\
S3-2 & 1.126 & 0.300 & 1.098 & 0.235 & 1.142 & 0.275 \\
S5-1 & 0.574 & 0.233 & 0.578 & 0.171 & 0.571 & 0.189 \\
S5-2 & 1.095 & 0.288 & 1.063 & 0.223 & 1.098 & 0.255 \\
O-1 & 0.620 & 0.189 & 0.638 & 0.152 & 0.642 & 0.192 \\
O-2 & 1.054 & 0.242 & 1.055 & 0.230 & 1.076 & 0.261 \\
\hline
\end{tabular}

The measured PGAs and peak response accelerations (PRAs) from the shaking tables tests are summarized in Table IV and compared in Fig. 21. The results obtained from the shaking table tests show that the PRAs are reduced to $0.25 \mathrm{~g}$ or less.

The measured peak ground displacements (PGDs) and peak response absolute displacements (PRADs) can be found in Table V. Compared to the PGDs, reduction of the PRADs can be observed in most of the sin wave input cases. However, to some sine wave inputs, the PRADs become larger than the PGDs. This is caused by shifting the center point of vibration due to the friction force. For the earthquake records, improvement cannot be found from the viewpoint of PRAD. Adding some dampers might be helpful to reduce PRADs. 

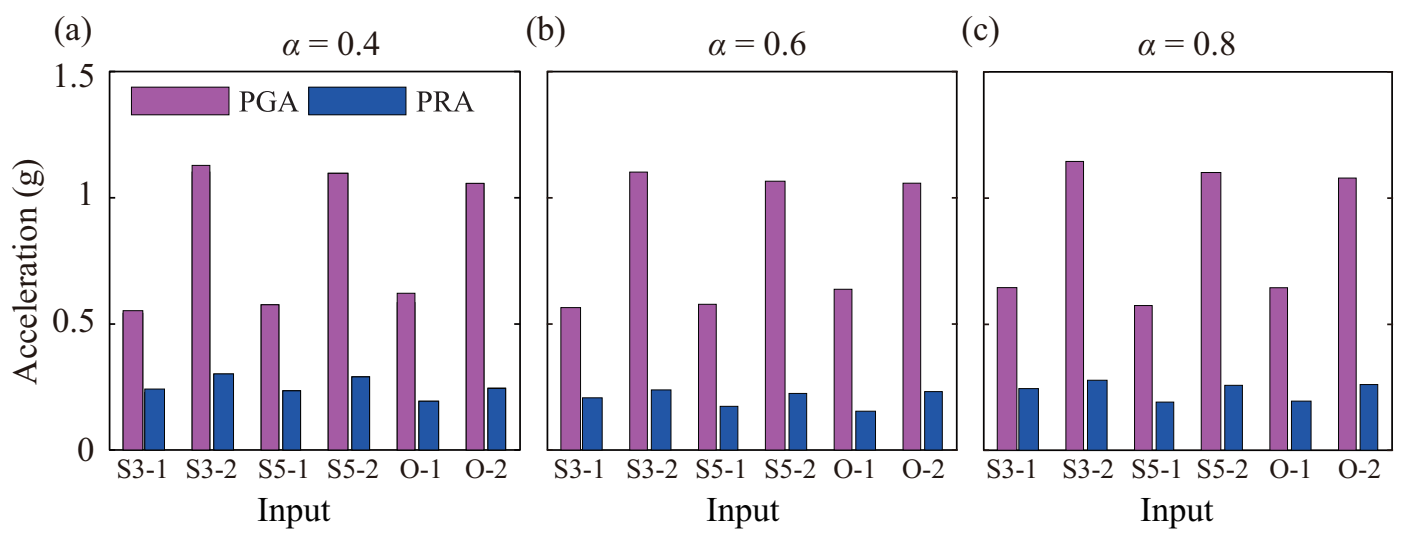

Figure 21. Comparisons of the measured PGAs and PRAs. (a) $\alpha=0.4$, (b) $\alpha=0.6$, (c) $\alpha=0.8$,

Table V. Comparisons between PGD and PRAD

\begin{tabular}{ccccccc}
\hline$\alpha$ & \multicolumn{2}{c}{0.4} & \multicolumn{2}{c}{0.6} & \multicolumn{2}{c}{0.8} \\
\hline Input & PGD $(\mathrm{mm})$ & PRAD $(\mathrm{mm})$ & PGD $(\mathrm{mm})$ & PRAD $(\mathrm{mm})$ & PGD $(\mathrm{mm})$ & PRAD $(\mathrm{mm})$ \\
\hline S3-1 & 12.85 & 8.80 & 12.75 & 4.15 & 12.90 & 6.40 \\
S3-2 & 24.85 & 9.70 & 24.75 & 6.05 & 24.70 & 8.75 \\
S5-1 & 4.85 & 13.3 & 4.80 & 1.55 & 4.85 & 10.00 \\
S5-2 & 9.05 & 2.85 & 8.95 & 1.70 & 9.00 & 2.40 \\
O-1 & 12.45 & 15.3 & 12.55 & 17.80 & 12.55 & 19.25 \\
O-2 & 20.70 & 26.05 & 20.75 & 24.90 & 20.80 & 22.55 \\
\hline
\end{tabular}

Time histories of the GA, RA, GD, RAD, and RRD to S5-2 and O-2 inputs for $\alpha=0.6$ are shown in Fig. 22. The figure shows that the RAs are reduced sufficiently during the entire excitation for both cases. The reduction of the RAD to S5-2 excitation and the amplification to O-2 excitation are observed in the plots of the time histories. And we can observe that due to the friction force, the response decays quickly after the excitation stops without installing additional dampers. Also, self-centering capacity of the prototype can be confirmed because the residue is not observed in both cases.

\subsection{Equivalent viscous damping}

To explore the damping effect caused by the friction force acting on the prototype in more detail, the equivalent viscous damping values for the cases of $\alpha=0.4,0.6$, and 0.8 are calculated as below. Let the loading and unloading forces be

$$
F_{1(\alpha)}=N_{1(\alpha)}+N_{2(\alpha)}+\frac{Q_{(\alpha)}}{2}-m g
$$

and

$$
F_{2(\alpha)}=N_{1(\alpha)}+N_{2(\alpha)}-\frac{Q_{(\alpha)}}{2}-m g
$$

Then the equivalent viscous damping for the prototype can be defined as

$$
\zeta_{\mathrm{eq},(\alpha)}=\frac{1}{4 \pi} \frac{2\left(F_{1(\alpha)}-F_{2(\alpha)}\right)}{F_{1(\alpha)} / 2}=\frac{Q_{(\alpha)}}{\pi\left(N_{1(\alpha)}+N_{2(\alpha)}+Q_{(\alpha)} / 2-m g\right)}
$$

It is worth noting that Eq. (31) shows that the equivalent viscous damping for the proposed device is independent of the displacement. Substituting the figures given in Table II and the mass for each 
(a)
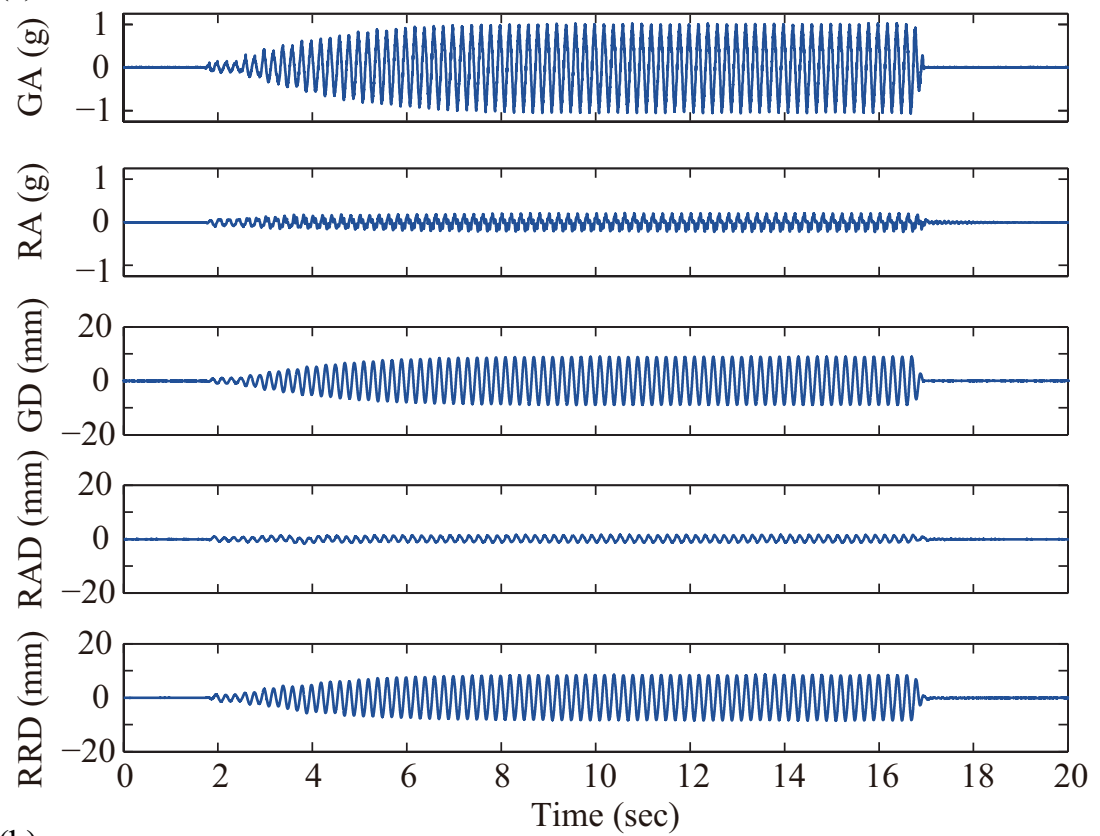

(b)
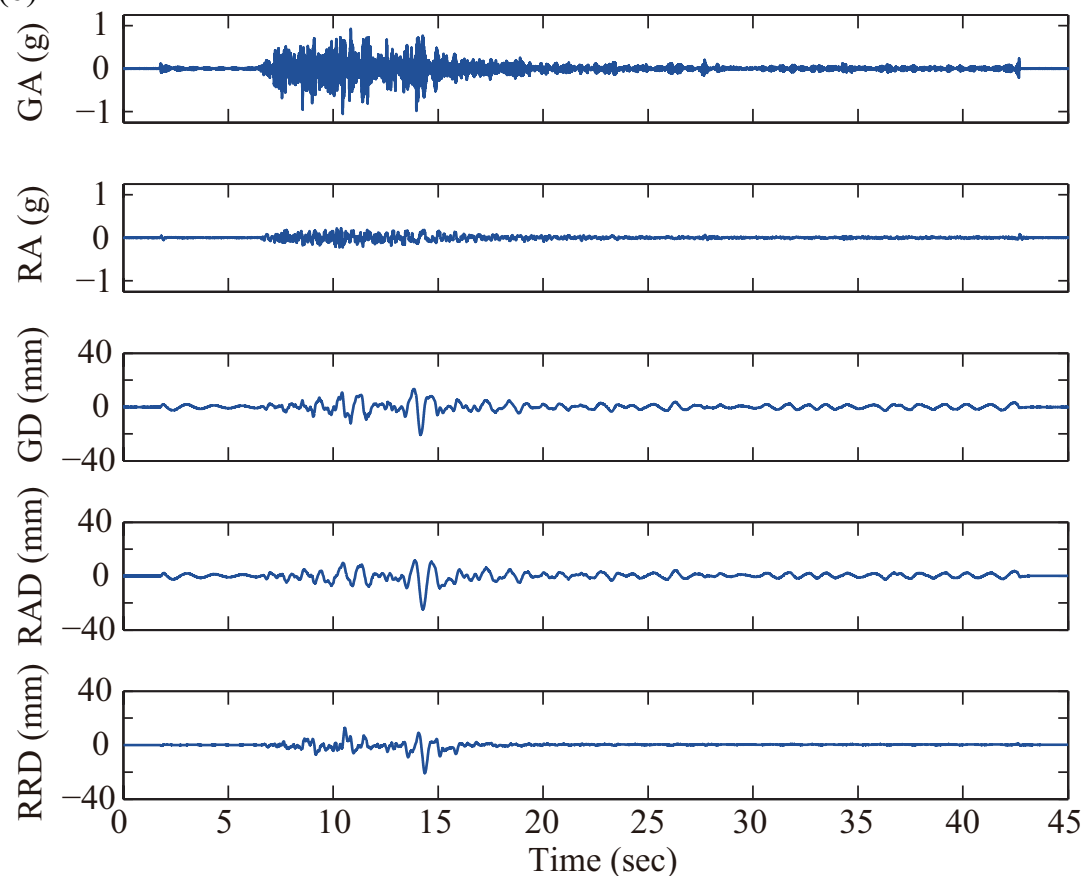

Figure 22. Time histories obtained from shaking table tests for $\alpha=0.6$. (a) S5-2, (b) O-2

case into Eq. (31) provides the equivalent damping for each case as $25.3 \%, 27.8 \%$, and $34.4 \%$, respectively. These numbers are relatively high compared to ordinary isolators. Thus it is shown that the prototype has enough damping capacity by the friction without additional dampers. Also the prototype satisfies the condition for self-centering function given by Eq. (27). Although the friction force is not easily controlled in an accurate way, considering these results, we can conclude that the prototype has appropriate damping values for isolators. 

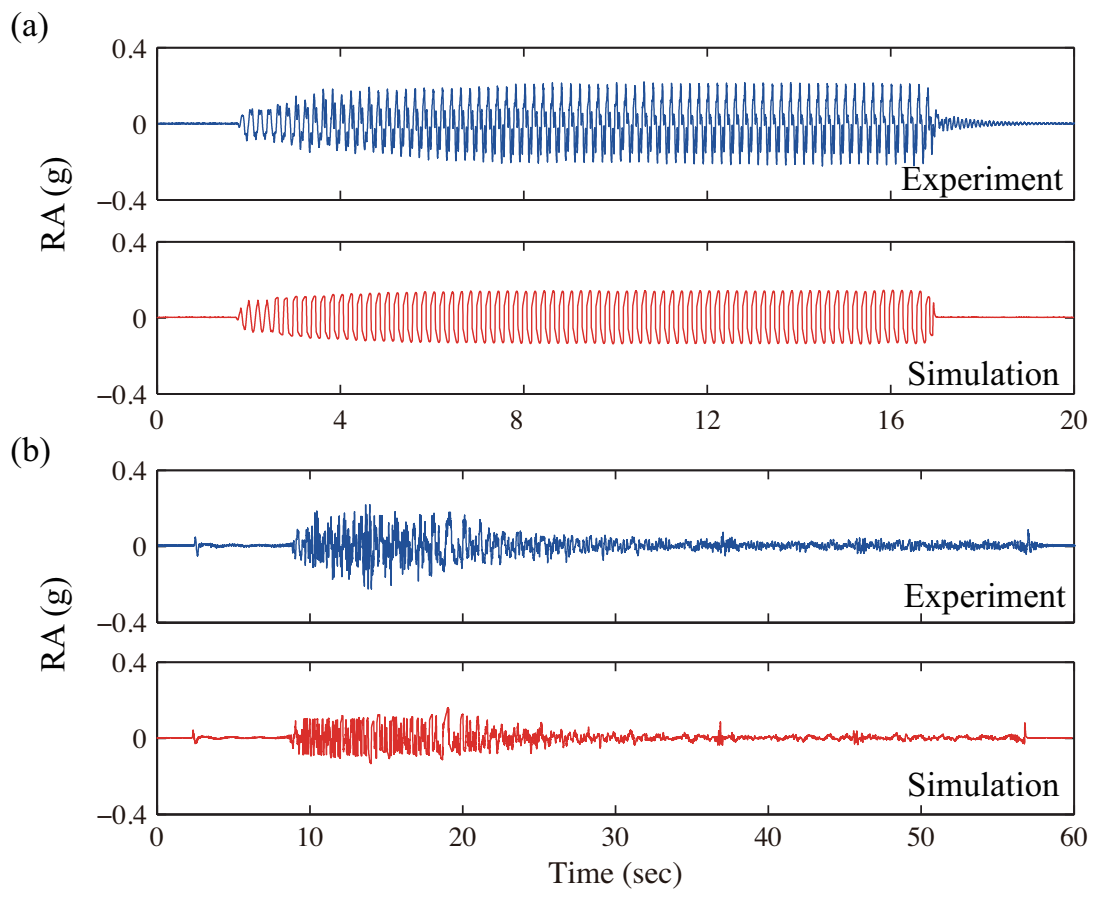

Figure 23. Comparisons of the time histories of the experiment and simulation (a) S5-2, (b) O-2

\section{NUMERICAL SIMULATIONS}

In this section, numerical simulation studies are implemented to compare with the experimental results. For the numerical simulations, the values of $N_{1(\alpha)}, N_{2(\alpha)}$, and $Q_{(\alpha)}$ obtained from the quasi-static loading tests given in Table II are used. And the mass $m$ is set to $15.82 \mathrm{~kg}$ for $\alpha=0.4$, $24.85 \mathrm{~kg}$ for $\alpha=0.6$, and $29.87 \mathrm{~kg}$ for $\alpha=0.8$, respectively, which are the same values as the experimental studies. The damping factor $c$ is assumed to be $0.01 \mathrm{~N} \mathrm{~s} / \mathrm{mm}$ in all cases. This value is determined by trial and error to fit the simulation results to the experimental data. To avoid singular points in the numerical simulations, the friction force $q$ is approximated by $q *$ defined as

$$
q *= \begin{cases}-Q & \text { if } \Delta \dot{w}<-Q / b \\ b \Delta \dot{w} & \text { if }-Q / b \leq \Delta \dot{w} \leq Q / b \\ Q & \text { if } Q / b<\Delta \dot{w}\end{cases}
$$

where the slope $b$ is determined as $1 \mathrm{~N} \mathrm{~s} / \mathrm{mm}$ in all cases by trial and error as well. The Runge-Kutta method embedded within MATLAB is used for the numerical simulations.

The time histories of the response accelerations obtained from the shaking table test and numerical simulation are compared. Fig. 23 shows the results when the S5-2 and O-2 excitations are input for the case of $\alpha=0.6$. We can observe that the simulation results are matched well with the experimental ones.

The PRAs obtained from the experiments and simulations are compared in Fig. 24. The red lines in the figures indicate the expected upper limits of the PRAs calculated by Eq. (25). As can be seen, most of the simulation values satisfy the expected upper limits. However, a few cases violate the expectation values. One of the possible causes is the damping effect. While most of the results obtained from the experiments exceed this limit values. Impacts occurred at the pins and shafts and the lack of the stiffness inside the device can be considered as possible causes. 
(a)

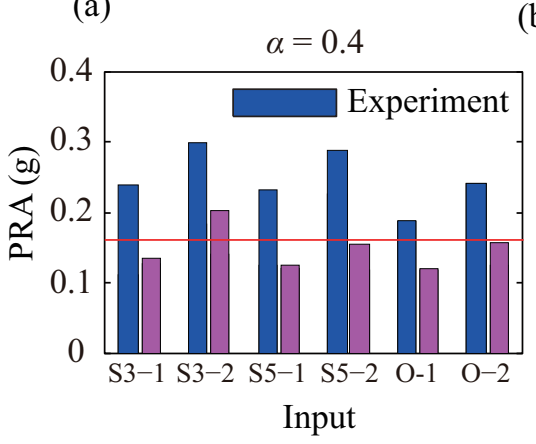

(b)

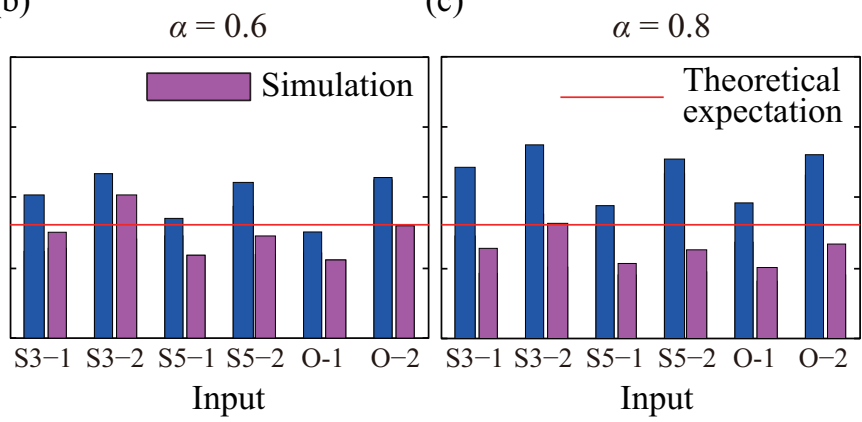

Figure 24. Comparisons of the PRAs (a) $\alpha=0.4$, (b) $\alpha=0.6$, (c) $\alpha=0.8$

\section{CONCLUSIONS}

This paper proposed the adjustable vertical vibration with the variable ellipse curve mechanism, in which the trajectories of the ellipse curve can be changed continuously without using complicated mechanisms. The features of the proposed device are as follows:

1. The amplification mechanism is realized through a horizontally placed plate. By doing this, the isolator can secure about twice as long stroke as the device with the mechanism in which vertically placed plates are used. In addition, the height of the device can be reduced significantly. Also, the horizontal mechanism using ellipse curve amplifies the restoring force of the constant-force springs at a constant rate.

2. Applying the principle of ellipsographs, the variable ellipse curve mechanism is realized. This mechanism can change the amplification factor of the device continuously and the device can adapt to various weight of target objects.

To verify the effectiveness of the proposed device for an object with a mass of several tens of kilograms, a prototype was made and shaking table tests and numerical simulations were implemented. We confirmed that the restoring force was scaled basically as expected by the ellipse curve mechanism through the quasi-static tests. In the shaking table tests, the measured PRAs were larger than the theoretically expected value, however, the errors were within about $15 \%$ to the various sine waves and scaled earthquake records. Thus we can conclude that the isolator with the proposed mechanism worked very well and the numerical simulations provided reliable values. In addition, by improving the device further, the experimental values can be expected to reduce close to the theoretically predicted value.

Finally, although the prototype used in this research was for relatively light objects, considering the loading capacity of commercially available constant-force springs and the proposed amplifying mechanism, the capacity of the proposed isolator could be increased up to several hundred kilograms for practical purposes.

\section{ACKNOWLEDGEMENT}

This research was supported by A-STEP program (AS2311091B), provided by the Japan Science and Technology Agency (JST). Mr. Nobutoshi Yoshida, a researcher of Kyoto University, helped with the shaking table and quasi-static loading tests. The authors gratefully acknowledge these supports.

\section{REFERENCES}

1. Ryan KL, Soroushian S, Maragakis EM, Sato E, Sasaki T, Okazaki T. Seismic simulation of an integrated ceilingpartition wall-piping system at e-defense. i: Three-dimensional structural response and base isolation. Journal of Structural Engineering 2016; 142(2):04015 130, doi:10.1061/(ASCE)ST.1943-541X.0001384. 
2. Furukawa S, Sato E, Shi Y, Becker T, Nakashima M. Full-scale shaking table test of a base-isolated medical facility subjected to vertical motions. Earthquake Engineering \& Structural Dynamics 2013; 42(13):1931-1949, doi:10.1002/eqe.2305.

3. Alabuzhev P, Rivin E. Vibration Protection And Measuring Systems With Quasi-Zero Stiffness. Applications of Vibration Series, Taylor \& Francis, 1989.

4. Ibrahim R. Recent advances in nonlinear passive vibration isolators. Journal of Sound and Vibration 2008; 314(3 5):371 - 452, doi:http://dx.doi.org/10.1016/j.jsv.2008.01.014.

5. Carrella A, Brennan M, Waters T, Shin K. On the design of a high-static-low-dynamic stiffness isolator using linear mechanical springs and magnets. Journal of Sound and Vibration 2008; 315(3):712 - 720, doi:http://dx.doi.org/10. 1016/j.jsv.2008.01.046. EUROMECH colloquium 483, Geometrically non-linear vibrations of structures.

6. Robertson WS, Kidner M, Cazzolato BS, Zander AC. Theoretical design parameters for a quasi-zero stiffness magnetic spring for vibration isolation. Journal of Sound and Vibration 2009; 326(1-2):88 - 103, doi:http: //dx.doi.org/10.1016/j.jsv.2009.04.015.

7. Platus DL. Negative-stiffness-mechanism vibration isolation systems. Proc. SPIE, vol. 1619, 1992; 44-54, doi: $10.1117 / 12.56823$.

8. Winterflood J, Blair D, Slagmolen B. High performance vibration isolation using springs in euler column buckling mode. Physics Letters A 2002; 300(2-3):122 - 130, doi:http://dx.doi.org/10.1016/S0375-9601(02)00258-X.

9. Kovacic I, Brennan MJ, Waters TP. A study of a nonlinear vibration isolator with a quasi-zero stiffness characteristic. Journal of Sound and Vibration 2008; 315(3):700 - 711, doi:http://dx.doi.org/10.1016/j.jsv.2007. 12.019. EUROMECH colloquium 483, Geometrically non-linear vibrations of structures.

10. Virgin L, Santillan S, Plaut R. Vibration isolation using extreme geometric nonlinearity. Journal of Sound and Vibration 2008; 315(3):721 - 731, doi:http://dx.doi.org/10.1016/j.jsv.2007.12.025. EUROMECH colloquium 483, Geometrically non-linear vibrations of structures.

11. Araki Y, Asai T, Masui T. Vertical vibration isolator having piecewise-constant restoring force. Earthquake Engineering \& Structural Dynamics 2009; 38(13):1505-1523, doi:10.1002/eqe.915.

12. Araki Y, Kawabata S, Asai T, Masui T. Response of vibration-isolated object to ground motions with intense vertical accelerations. Engineering Structures 2011; 33(12):3610 - 3619, doi:http://dx.doi.org/10.1016/j.engstruct.2011.07. 025.

13. Araki Y, Asai T, Kimura K, Maezawa K, Masui T. Nonlinear vibration isolator with adjustable restoring force. Journal of Sound and Vibration 2013; 332(23):6063 - 6077, doi:http://dx.doi.org/10.1016/j.jsv.2013.06.030.

14. Araki Y, Kimura K, Asai T, Masui T, Omori T, Kainuma R. Integrated mechanical and material design of quasi-zerostiffness vibration isolator with superelastic cu-al-mn shape memory alloy bars. Journal of Sound and Vibration 2015; 358:74 - 83, doi:http://dx.doi.org/10.1016/j.jsv.2015.08.018.

15. Sun X, Jing X, Xu J, Cheng L. Vibration isolation via a scissor-like structured platform. Journal of Sound and Vibration 2014; 333(9):2404 - 2420, doi:http://dx.doi.org/10.1016/j.jsv.2013.12.025.

16. Sun X, Jing X. Analysis and design of a nonlinear stiffness and damping system with a scissor-like structure. Mechanical Systems and Signal Processing 2016; 66 - 67:723 - 742, doi:http://dx.doi.org/10.1016/j.ymssp.2015. 05.026.

17. Cardone D. Re-centring capability of flag-shaped seismic isolation systems. Bulletin of Earthquake Engineering 2012; 10(4):1267-1284, doi:10.1007/s10518-012-9343-1.

18. Lee GC, Ou YC, Niu T, Song J, Liang Z. Characterization of a roller seismic isolation bearing with supplemental energy dissipation for highway bridges. Journal of Structural Engineering 2010; 136(5):502-510, doi:10.1061/ (ASCE)ST.1943-541X.0000136.

19. Wang SJ, Hwang JS, Chang KC, Shiau CY, Lin WC, Tsai MS, Hong JX, Yang YH. Sloped multi-roller isolation devices for seismic protection of equipment and facilities. Earthquake Engineering \& Structural Dynamics 2014; 43(10):1443-1461, doi:10.1002/eqe.2404. EQE-13-0084.R3.

20. Eatherton MR, Hajjar JF. Residual drifts of self-centering systems including effects of ambient building resistance. Earthquake Spectra 2011; 27(3):719-744, doi:10.1193/1.3605318. 